\title{
HeAd AND NeCK Symposium
}

\section{Imaging in laryngeal cancers}

\author{
Varsha M Joshi, Vineet Wadhwa1', Suresh K Mukherji² \\ Vijaya Diagnostics, ' $A$ pollo Hospitals, Hyderabad, India, ${ }^{2}$ University of Michigan, Ann Arbor, MI, USA
}

Correspondence: Varsha Joshi, Senior Consultant, Vijaya Diagnostics, Hyderabad, India. Email: drjoshivarsha@gmail.com

\begin{abstract}
Imaging plays an important complementary role to clinical examination and endoscopic biopsy in the evaluation of laryngeal cancers. A vast majority of these cancers are squamous cell carcinomas (SCC). Cross-sectional imaging with contrast-enhanced computed tomography (CT) and magnetic resonance (MR) imaging allows excellent depiction of the intricate anatomy of the larynx and the characteristic patterns of submucosal tumor extension. CT, MRI and more recently PET-CT, also provide vital information about the status of cervical nodal disease, systemic metastases and any synchronous malignancies. Additionally, certain imaging-based parameters like tumor volume and cartilaginous abnormalities have been used to predict the success of primary radiotherapy or surgery in these patients. Integration of radiological findings with endoscopic evaluation greatly improves the pretherapeutic staging accuracy of laryngeal cancers, and significantly impacts the choice of management strategies in these patients. Imaging studies also help in the post-therapeutic surveillance and follow-up of patients with laryngeal cancers. In this article, we review the currently used laryngeal imaging techniques and protocols, the key anatomic structures relevant to tumor spread and the characteristic patterns of submucosal extension and invasion of laryngeal cancer. The role of CT, MRI and PET-CT in the evaluation of patients with laryngeal SCC and the impact of imaging findings on prognosis and clinical management is also discussed.
\end{abstract}

Key words: CT; imaging; laryngeal cancer; MRI; post-treatment; staging

\section{Introduction}

Cancers of the larynx constitute about $25 \%$ of all head and neck malignancies. They commonly present in adults between 50 and 70 years and show a strong male predominance. ${ }^{[1]}$ Over $90 \%$ of these cancers are squamous cell carcinomas (SCC). Tobacco smoking and alcohol consumption are important risk factors for laryngeal SCC. ${ }^{[1,2]}$ Patients with laryngeal SCC have a higher risk for synchronous malignancies arising from the lung and upper aerodigestive tract. ${ }^{[3]}$

Majority of these SCCs are readily identified at endoscopy. Integration of endoscopic findings with cross-sectional imaging to assess the submucosal and loco-regional extent of the SCC improves the T staging accuracy and influences

\begin{tabular}{|l|l|}
\hline \multicolumn{2}{|c|}{ Access this article online } \\
\hline Quick Response Code: & \\
\hline & Website: \\
\hline & www.ijri.org \\
\cline { 2 - 3 } & DOI: \\
\hline
\end{tabular}

the treatment decisions in these patients. Imaging also provides information about the nodal disease, systemic metastases, any synchronous tumors and recurrent disease. $^{[4]}$

This article reviews the standard imaging protocols to evaluate the larynx and the key anatomical structures relevant to tumor spread, and illustrates the characteristic patterns of submucosal spread of this SCC. The influence of imaging findings on prognosis and choice of treatment options in these patients is also described.

\section{Imaging Protocols}

CT

Evaluation of laryngeal SCC requires a contrast CT study of the neck. Excellent images of the neck are obtained using a multidectector CT (MDCT) following the injection of an iodinated contrast agent (total dose 35-40 g). The contrast may be hand injected or an automated power injector may be used, in which case sufficient delay should elapse before scan acquisition begins. The patient lies in supine position, breathing quietly and is asked to refrain from coughing or swallowing. Axial scanning is performed from the skull base to the aortic arch with the acquisition plane parallel to 
the plane of hyoid bone, to obtain scans parallel to the true vocal cords. The raw axial image dataset is reconstructed with a section thickness of as little as $0.75 \mathrm{~mm}$ to obtain high quality sagittal and coronal reformatted images. A $512 \times 512$ matrix is used with a small field of view (FOV) between 16 and $20 \mathrm{~cm}$. All images are reviewed in soft tissue and bone windows. An additional examination for better assessment of the tumor in laryngeal ventricle, anterior commisure and aryepiglottic folds may be done with e-phonation. ${ }^{[5,6]}$

\section{MRI}

A high field MRI scanner using a dedicated neck coil is preferred. A combination of multiplanar noncontrast T1-weighted, T2-weighted and T2-weighted fat saturation images with postcontrast T1 fat-suppressed images are routinely used. It is important to take the T1 and $\mathrm{T} 2$ sections at the same levels. A section thickness of $4 \mathrm{~mm}$ is preferred with an interslice gap of $0-1 \mathrm{~mm}$. The entire examination takes about 30 minutes, and the patient is asked to refrain from coughing and swallowing during the acquisition.

The choice of imaging modality is subject to the availability of the CT or MR scanner and the expertise in interpretation of the scans, as also the ability of the patient to tolerate an MR examination. In most institutions, $\mathrm{CT}$ is the preferred imaging method for evaluating laryngeal SCC and MRI is used as a complementary problem-solving tool when CT does not provide all the information prior to therapy. ${ }^{[7]}$

\section{Key Anatomical Features of the Larynx}

A clear understanding of the laryngeal anatomy is fundamental to the interpretation of CT and MRI scans of patients with laryngeal SCC. A detailed review of the anatomy ${ }^{[8]}$ is beyond the scope of this article. A few key anatomical features are discussed below ${ }^{[8,9]}$ [Figures 1-4].

\section{Laryngeal cartilages}

The larynx extends from the tip of epiglottis to the inferior margin of the cricoid cartilage. The epiglottis, thyroid, cricoid and the paired arytenoid cartilages are the four principal laryngeal cartilages [Figure 1].

The epiglottis is the superiormost, midline leaf-shaped cartilage. It has a free margin and a fixed portion (stem). The hyoepiglottic ligament attaches the free epiglottic margin to the hyoid bone and the thyroepiglottic ligament attaches the epiglottic stem to the inner surface of the thyroid cartilage at a caudal level. The free margin of the epiglottis is closely related to the base of tongue - the median glosso-epiglottic fold runs over the hyoepiglottic ligament and projects above the level of the hyoid. Hence, on serial axial CT or MR images of the neck, the free

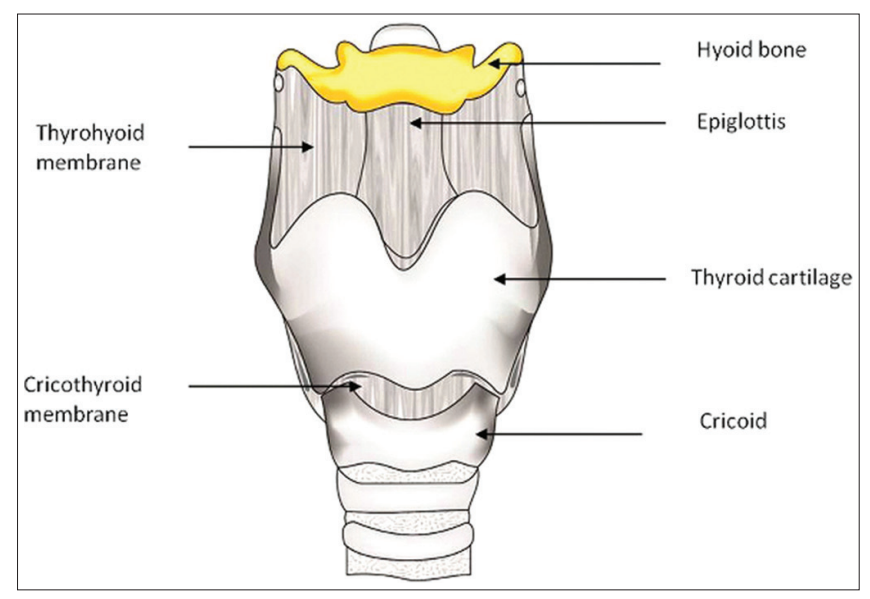

Figure 1A: Normal anatomy of larynx. Frontal view

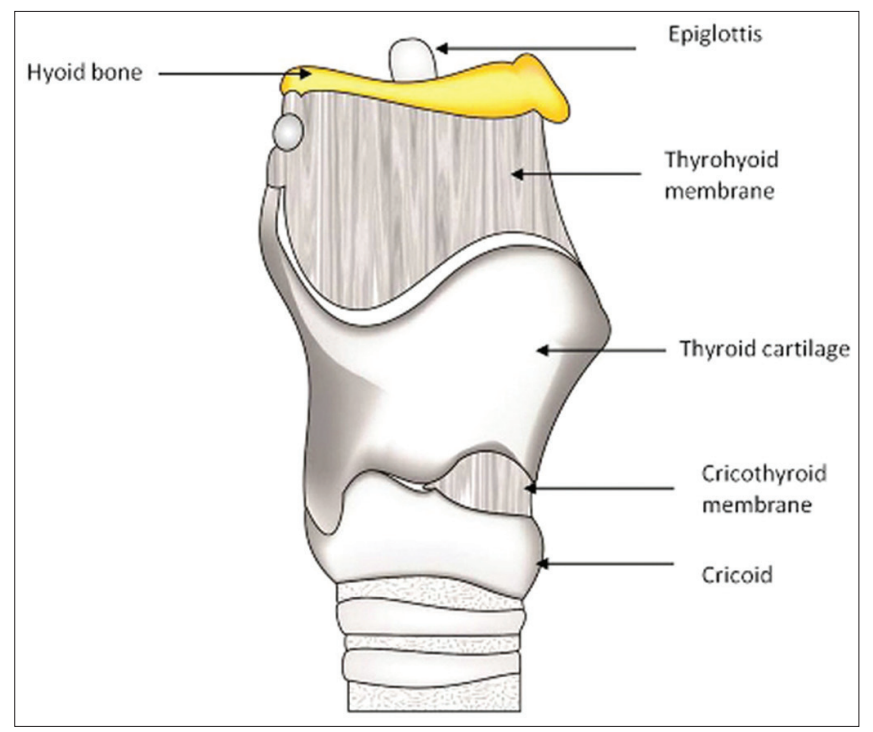

Figure 1B: Normal anatomy of larynx. Lateral view

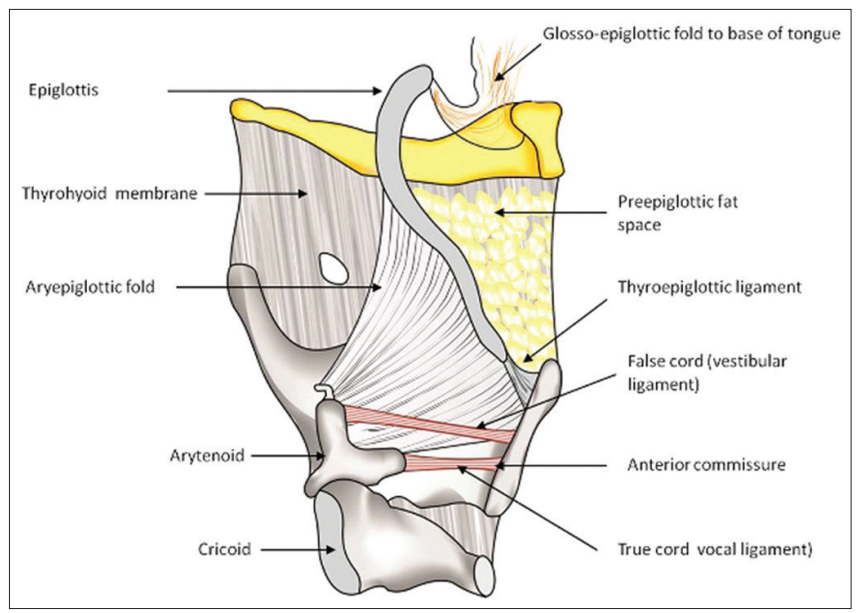

Figure 1C: Normal anatomy of larynx. Lateral view with one thyroid lamina removed

epiglottic margin appears before the hyoid is visualised. The thyroid cartilage has two laminae fused anteriorly, 


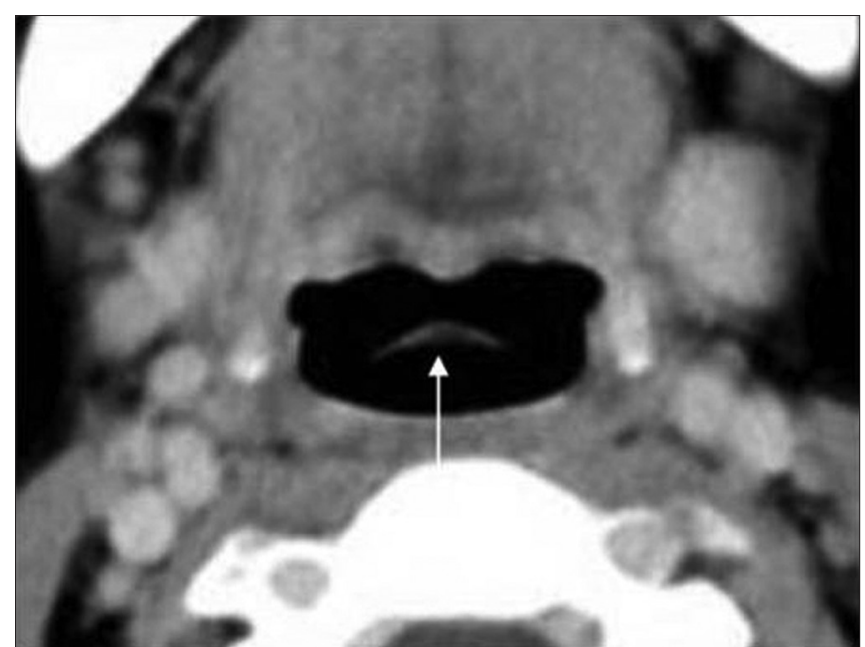

Figure 2A: Normal anatomy of larynx on axial contrast CT images supraglottis. Axial contrast CT image shows the tip of the epiglottis in the midline (thin arrow)

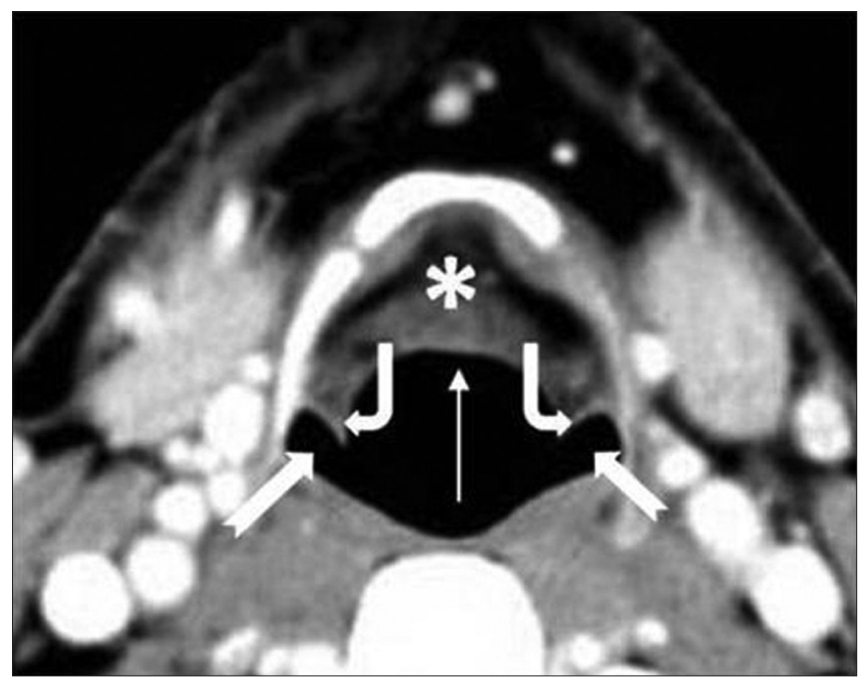

Figure 2C: Normal anatomy of larynx on axial contrast CT images supraglottis. The preepiglottic space (white asterisk) seen anterior to the epiglottis (thin arrow). Note the aryepiglottic folds (bent arrows) and the piriform sinuses (notched arrows)

and enlarged posteriorly, to form the superior and inferior cornua. The superior cornua provide attachment to the thyrohyoid ligament and the inferior cornua articulate medially with the sides of the cricoid at the cricothyroid joint. The cricoid is the inferiormost ring-shaped laryngeal cartilage and the foundation of the larynx. The paired arytenoid cartilages sit along the upper margin of the cricoid lamina, forming the cricoarytenoid joints. The vertical height of the arytenoid spans the laryngeal ventricle. The apex of the arytenoid attaches the vestibular ligament and corresponds to the level of the false cords. The base of the arytenoids projects the vocal processes anteriorly, that attach the vocal ligament running along the inner margin of true vocal cords.

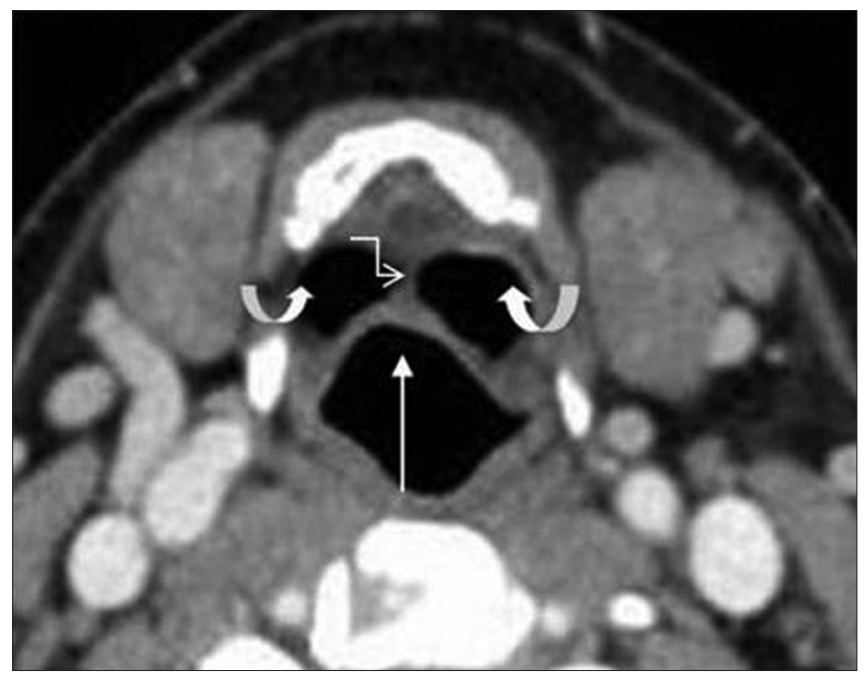

Figure 2B: Normal anatomy of larynx on axial contrast CT images - supraglottis. Paired valleculae (curved arrows) on either sides of hyoepiglottic ligament (elbow arrow). Epiglottis is seen in the midline (thin arrow)

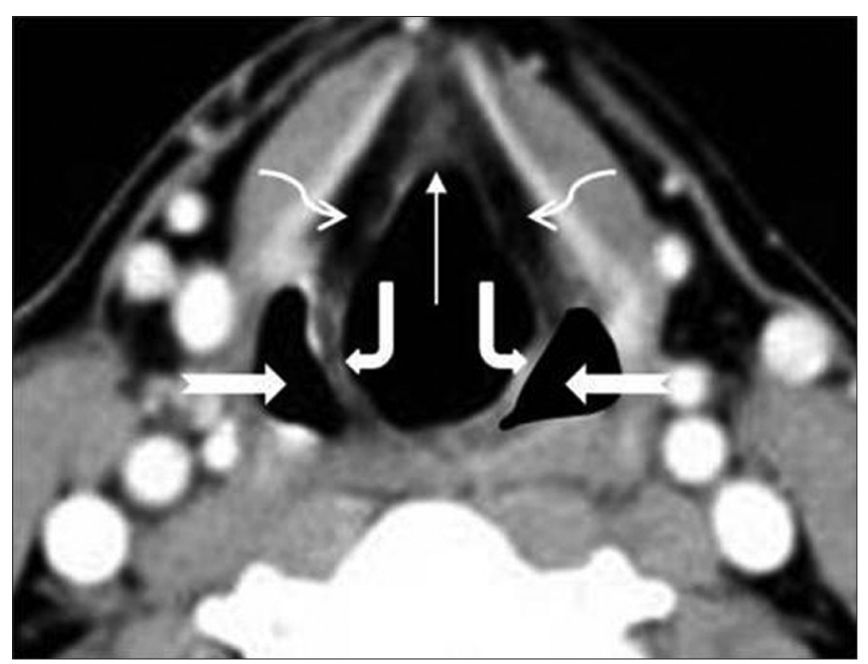

Figure 2D: Normal anatomy of larynx on axial contrast CT images - supraglottis. The stem of the epiglottis is seen attaching to the inner surface of the thyroid cartilage in the midline (thin arrow). The aryepiglottic folds (bent thick arrows), piriform sinuses (notched arrows) and paraglottic spaces (curved elbow arrows) are seen

The imaging appearance of these cartilages depends on whether or not they are ossified. The epiglottis and the vocal process of arytenoids are fibrocartilages that do not ossify. Nonossified cartilages have soft tissue attenuation on CT and intermediate signal intensity on T1-weighted (T1W) and T2-weighted (T2W) images. The thyroid, cricoid and arytenoid are hyaline cartilages that show progressive ossification with age. On $\mathrm{CT}$, the ossified cartilages have hyperattenuating inner and outer margins with low attenuation of the medullary cavity. On MRI, the ossified cortical margins are of low signal and the fat-filled medullary cavity is of high signal on T1W and $\mathrm{T} 2 \mathrm{~W}$ images. 


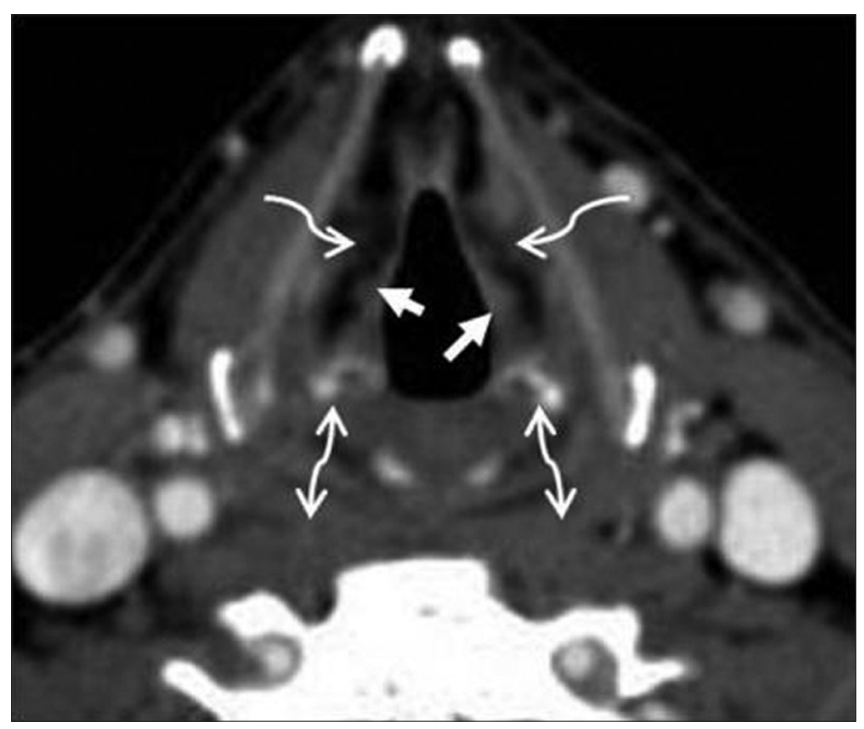

Figure 2E: Normal anatomy of larynx on axial contrast CT images supraglottis. The tip of the arytenoid cartilages (double headed arrows) and the false cords (small arrows) are seen. The paraglottic spaces (curved elbow arrows)are seen deep to the false cords. This section represents the superior margin of the laryngeal ventricular complex

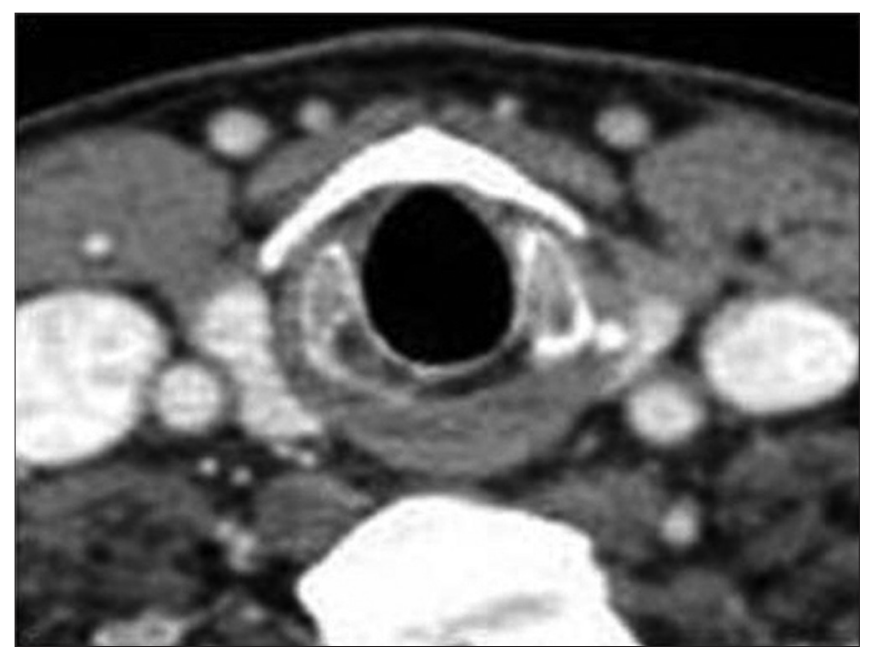

Figure 2G: Normal anatomy of larynx on axial contrast CT images subglottis. Axial section through the subglottis shows the cricoid ring

\section{Laryngeal Ventricular Complex (LVC)}

This is the key component in organizing the larynx into the supraglottis, glottis and subglottis [Table 1]. It comprises the false cords, true cords and intervening laryngeal ventricle.

The LVC is best identified on coronal images [Figure 4]; the ventricle itself is seen as a small air-filled outpouching between the false and true cords. On axial images, the superior margin of the LVC is defined by a section through the apex of the arytenoids, the false cords and the fat-filled paraglottic spaces deep to the false cords [Figure 2E]. The inferior margin is defined by a section through the true cords with the thyroarytenoid muscle and the cricoarytenoid joint [Figure 2F].

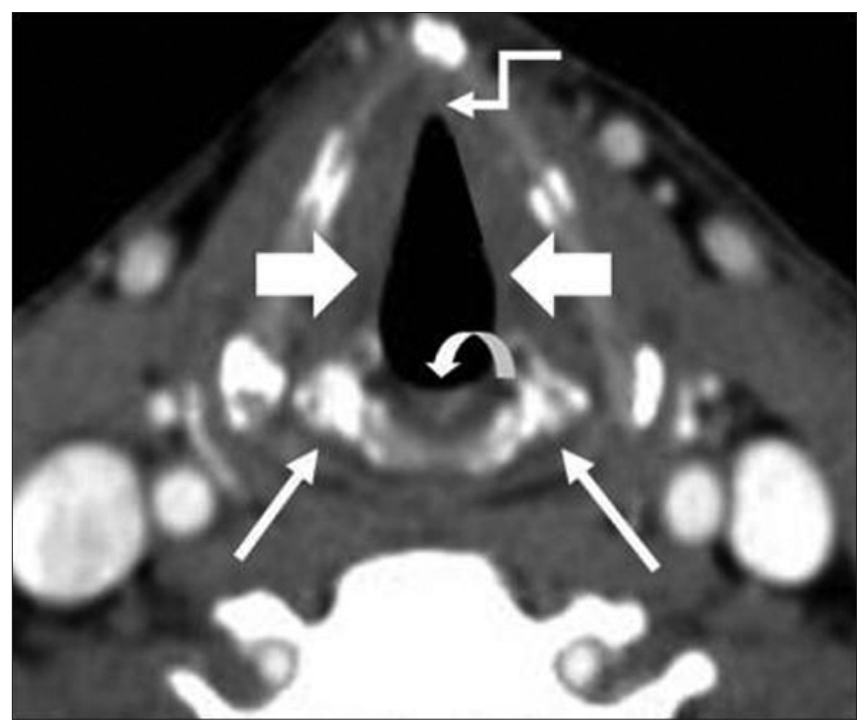

Figure 2F: Normal anatomy of larynx on axial contrast CT images - glottis. The cricoarytenoid joints (straight arrows) are seen. The thyroarytenoid muscle forms the bulk of the true vocal cords (block arrows) at this level. Note the anterior commissure (elbow arrow) and the posterior commissure (curved arrow)

\section{Anterior and Posterior Commissure}

The anterior commissure is the midline anterior meeting point of the true vocal cords. It comprises of the anterior cord, the anterior junction of the two vocal cords, the thyroid cartilage and the Broyle's ligament, a fibrous structure connecting the vocal ligaments to the cartilage. There is no perichondrium at that point, so the fibers extend directly from the vocal ligament into the cartilage. The posterior commissure is the mucosal surface on the anterior surface of the cricoid cartilage between the arytenoid cartilages. Both the commissures are seen very well on the axial images [Figure 2F].

\section{The Paraglottic Space (PLS) and the Pre-Epiglottic Space (PES)}

The PGS is located deep to the mucosal surfaces of the true and false cords and bound laterally by the thyroid and cricoid cartilages and is best seen on axial CT and MR sections through the supraglottis (2C-E), where it is entirely composed of fat. It extends caudally upto the undersurface of the true vocal cords. The entire extent is clearly demonstrated on coronal images [Figure 4]. The PGS is continuous with the extralaryngeal soft tissues between the thyroid and cricoid cartilages antero-laterally; an important pathway for extralaryngeal tumor spread. The pre-epiglottic space (PES) is a fat-filled space, rich in lymphatics. It is bound superiorly by the hyoepiglottic ligament, anteriorly by the thyrohyoid membrane, inferiorly by the thyroepiglottic ligament and posteriorly by the epiglottis. The PES and PGS communicate with each other superiorly. Sagittal images are best suited to delineate the entire extent of the PES [Figure 3]; 


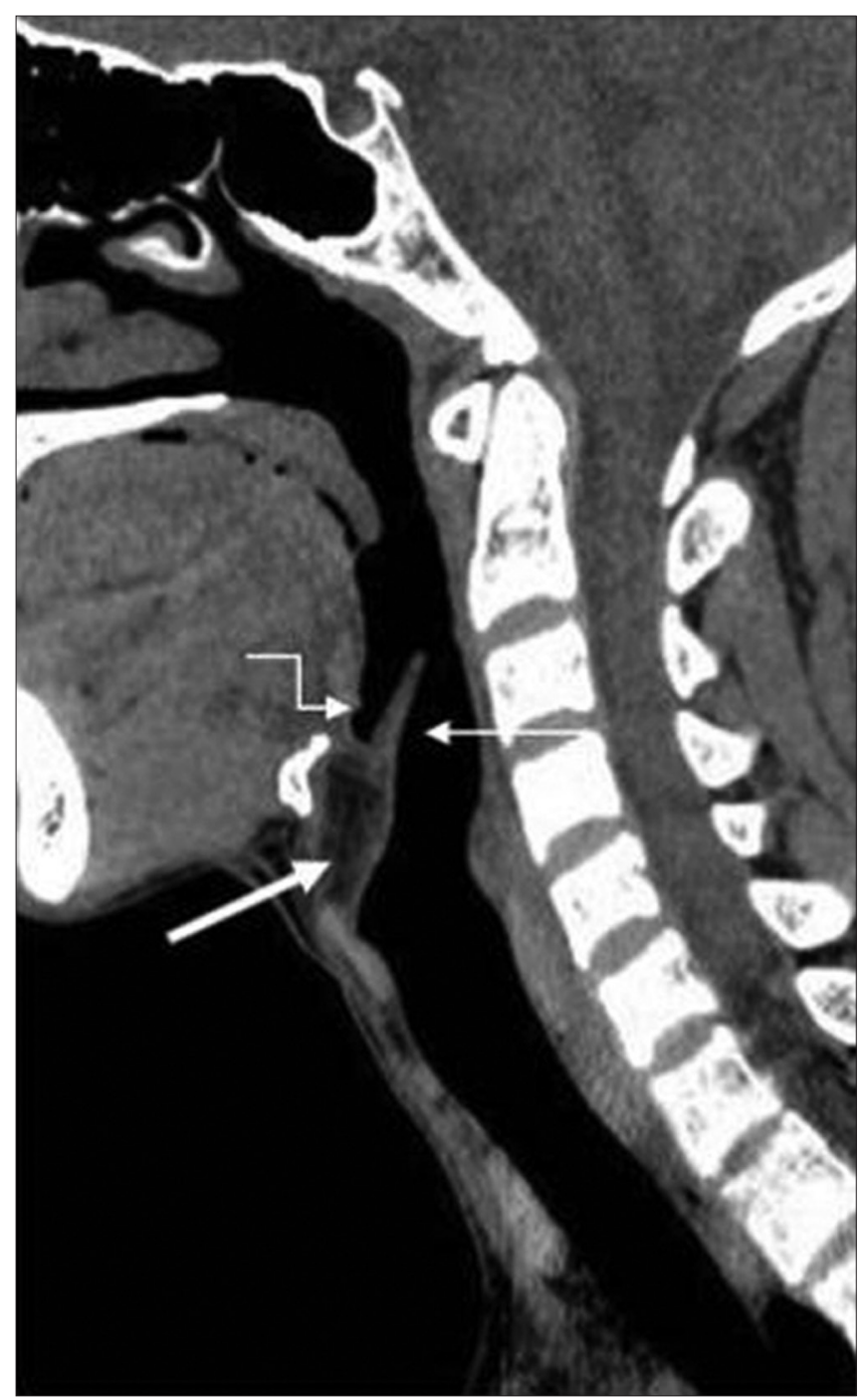

Figure 3A: Sagittal CT image through the larynx. Sagittal CT reformation shows the epiglottis (thin arrow) and the preepiglottic fat space (thick arrow). Note the close relationship of the base of tongue (elbow arrow) with the epiglottis

however, axial images also provide good delineation of the same [Figure 2C-E].

\section{Tumor Origin and Characteristic Patterns of Spread (T staging)}

Clinical examination followed by endoscopy is always the first step in T staging of laryngeal SCC. CT and MRI are performed to define the submucosal extent and deeper margins of the tumor. Small and superficial mucosal tumors may not be appreciated at CT or MRI and hence, it is mandatory that an endoscopy is done prior to any imaging study. Integration of cross-sectional imaging with endoscopy findings significantly improves the accuracy of $\mathrm{T}$ staging. Zbaren, et al. reported the accuracy of clinical $\mathrm{T}$ staging alone for laryngeal SCC to be $57.5 \%$, but as high as $80 \%$ when combined with contrast-enhanced CT. ${ }^{[9]}$

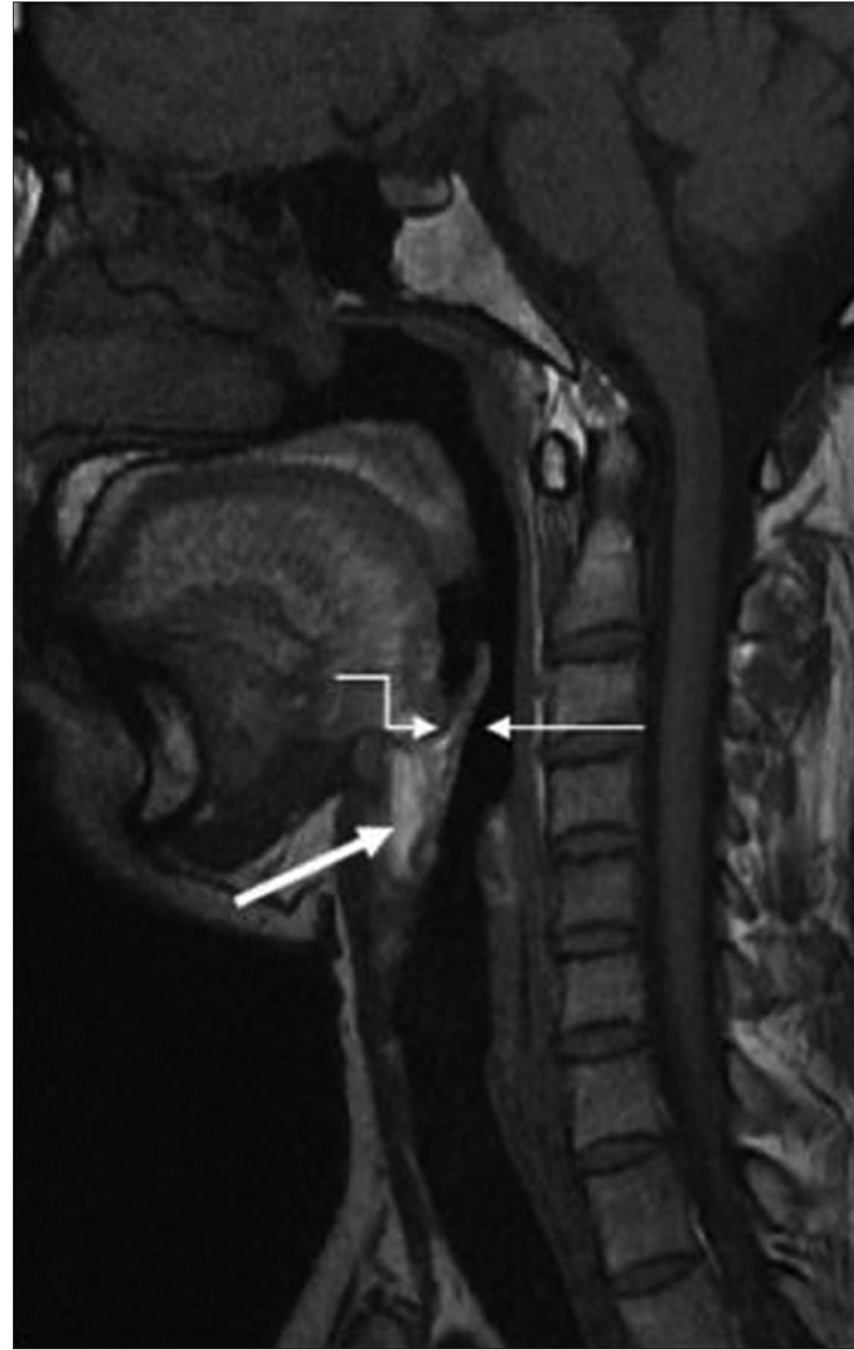

Figure 3B: Sagittal T1W MR image through the larynx. Sagittal T1W MRI shows the epiglottis (thin arrow) and the preepiglottic fat space (thick arrow). Note the close relationship of the base of tongue (elbow arrow) with the epiglottis

The TNM classification laid down by the American Joint Commission on Cancer (AJCC) is universally accepted for staging laryngeal cancer ${ }^{[10]}$ [Table 2]. This classification incorporates all information available prior to treatment, including the clinical examination, endoscopy, endoscopic biopsy and cross-sectional imaging. The guidelines rely heavily on the use of cross-sectional imaging for the $\mathrm{T}$ staging; however, no recommendation is made regarding the preference of one technique over the other.

The general radiological criteria used for tumor involvement include asymmetric soft tissue prominence or thickening, abnormal contrast enhancement, a bulky mass, obliteration of the normal fat planes and spaces, or a combination of these. ${ }^{[11]}$

Supraglottic SCC

Approximately $30 \%$ of all laryngeal cancers arise in the supraglottis. They often present in advanced stages, because symptoms (hoarseness, due to vocal cord 
Table 1: Laryngeal subdivisions

\begin{tabular}{lll}
\hline Subdivision & Extent & Contents \\
\hline Supraglottis & Tip of epiglottis to laryngeal ventricle & Epiglottis \\
& & False cords \\
& Aryepiglottic \\
& folds \\
& Arytenoid \\
& & cartilages \\
& & Preepiglottic \\
& & space \\
& & Paraglottic \\
& & space \\
Glottis & & Vestibule \\
& & True vocal \\
& From laryngeal ventricle to an & cords \\
& imaginary plane $1 \mathrm{~cm}$ below this level & Anterior \\
& & commissure \\
& & Posterior \\
Subglottis & From undersurface of true cord to & \\
& inferior surface of cricoid cartilage & \\
& &
\end{tabular}

Table 2: T- staging of Laryngeal Cancers (according to AJCC)

\section{Supraglottic SCC}

T1 Tumor confined to one supraglottic subsite with normal vocal cord mobility

T2 Tumor invades mucosa in more than one supraglottic subsite, without cord fixation

T3 Tumor limited to the larynx, with vocal cord fixation and/or invasion of postcricoid area or preepiglottic space

T4A Resectable: Tumor invading through the thyroid cartilage and/or other extralaryngeal tissues (trachea, cervical soft tissues, strap muscles, thyroid, esophagus)

T4B Unresectable: Tumor invading prevertebral space, encasing the carotid artery, or invading mediastinal structures

Glottic SCC

T1 Tumor limited to vocal cord (s), with normal mobility (may involve anterior or posterior commissure

T1A Limited to one cord

T1B Involving both cords

T2 Tumor extension to supra and/or subglottis with impaired vocal cord mobility

T3 Tumor limited to the larynx, with vocal cord fixation and/or invasion of paraglottic space and/or inner cortex of thyroid cartilage

T4A Resectable: Tumor invading through the thyroid cartilage and/or other extralaryngeal tissues (trachea, cervical soft tissues, deep extrinsic muscles of tongue, strap muscles, thyroid, esophagus)

T4B Very advanced local disease: Tumor invading prevertebral space, encasing the carotid artery, or invading mediastinal structures

\section{Subglottic SCC}

T1 Tumor limited to subglottis

T2 Tumor extending to vocal cord (s), with normal or impaired mobility

T3 Tumor limited to larynx with fixed vocal cords

T4A Resectable: Tumor invading cricoids and/or thyroid cartilage and/or invading tissues beyond the larynx (trachea, cervical soft tissues, deep extrinsic muscles of tongue, strap muscles, thyroid, esophagus)

T4B Unresectable: Tumor invading prevertebral space, encasing the carotid artery, or invading mediastinal structures

involvement) do not occur until late. Due to the rich lymphatic network of the supraglottis, nodal disease (level

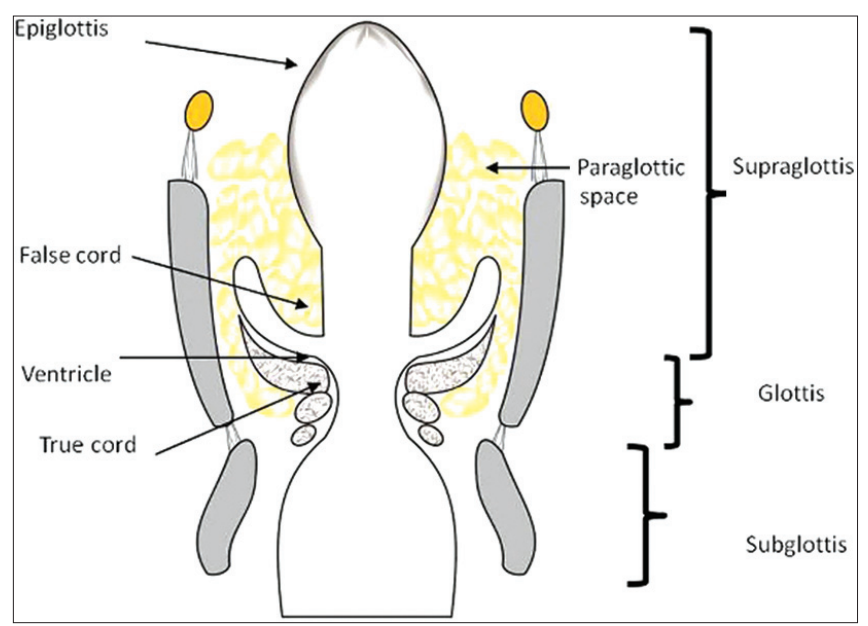

Figure 4A: Coronal image though the larynx. Line diagram shows complete extent of the paraglottic space and the laryngeal ventricle complex in the coronal plane. Note the laryngeal subdivisions

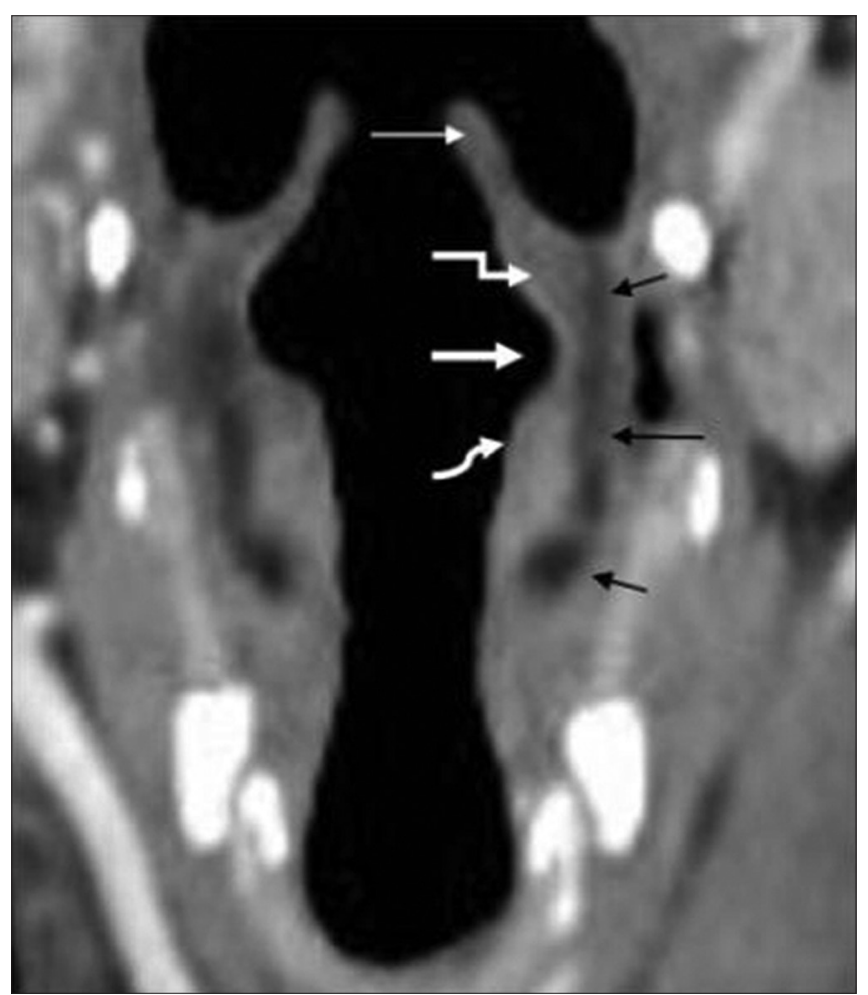

Figure 4B: Coronal CT image through the larynx. Coronal CT image shows the epiglottis (thin arrow), false cord (elbow arrow), laryngeal ventricle (thick arrow), true cord (curved elbow arrow) and paraglottic space (asterisk)

II and III) is a frequent finding in these patients. The overall 5-year survival rate is $75 \%$. $^{[12]}$

Supraglottic SCC may arise in the anterior compartment (epiglottis) or the postero-lateral compartment (aryepiglottic fold and false cords).

\section{a. Epiglottic SCC}

These are anterior midline cancers that primarily invade 


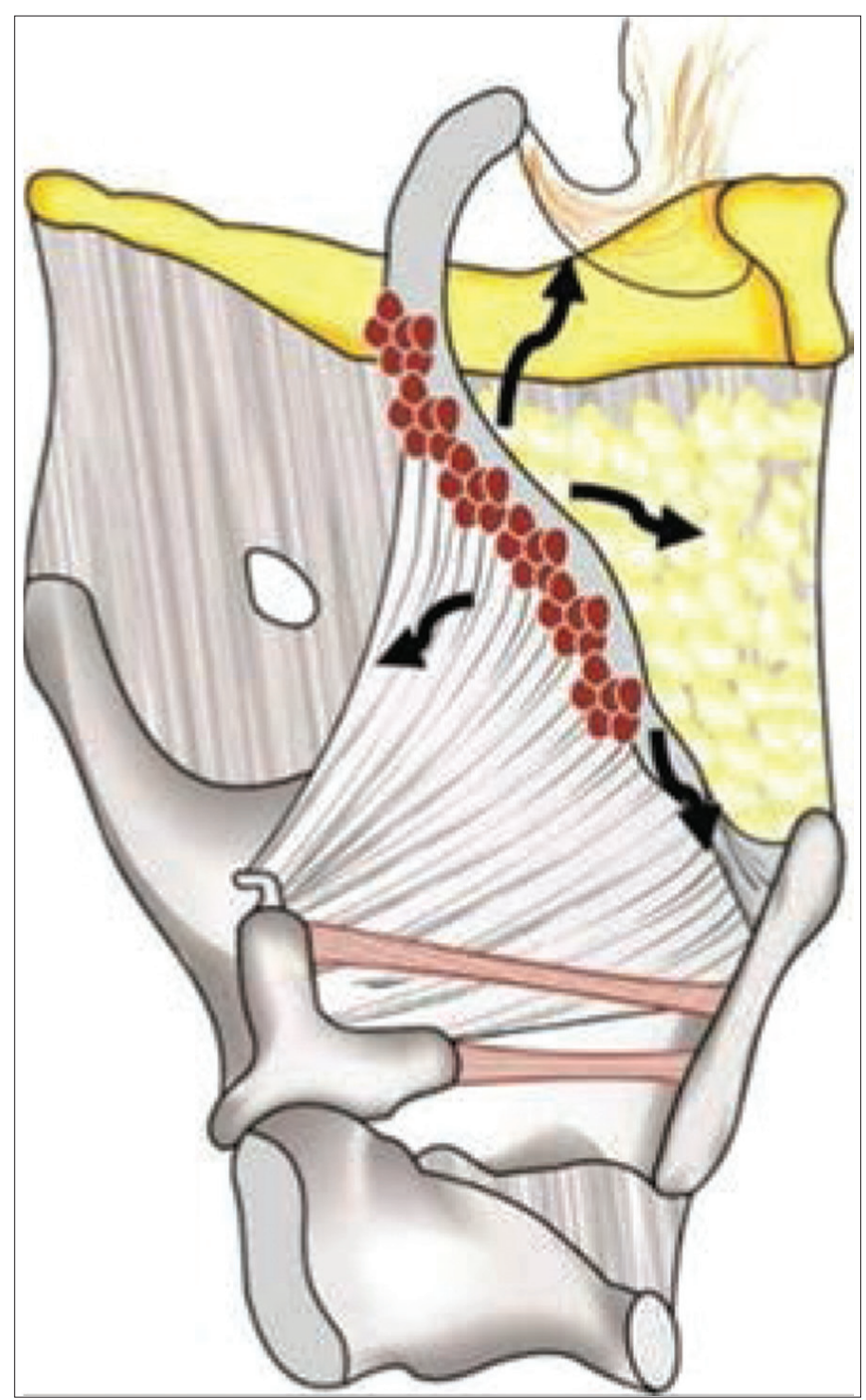

Figure 5A: Supraglottic mass arising from the epiglottis. Sagittal line diagram shows the pathways of spread (thick black arrows) of epiglottic mass (red colour)

into the PES [Figures 5 and 6]. While the SCCs arising from the mobile portion of the epiglottis may spread from the PES further into the base of tongue and laterally into the PGS, those arising from the stem often invade the low PES and via the anterior commissure, reach the glottis or subglottis [Figure 5]. The primary sign of PES invasion at imaging is replacement of the normal fat by abnormal enhancing soft tissue [Figures 6,7,9,18A and 19B]. The sensitivity of CT and MRI to detect invasion of the PES is $100 \%$ and the corresponding specificities are $93 \%$ and $84-90 \% \cdot{ }^{[9,13]}$

\section{b. Aryepiglottic fold (AE fold) SCC}

These cancers present as exophytic or infiltrative masses. They expand the AE fold and spread into the PGS. They may spread further anteriorly into the PES or posteriorly to invade the piriform sinus [Figures 8 and 9].

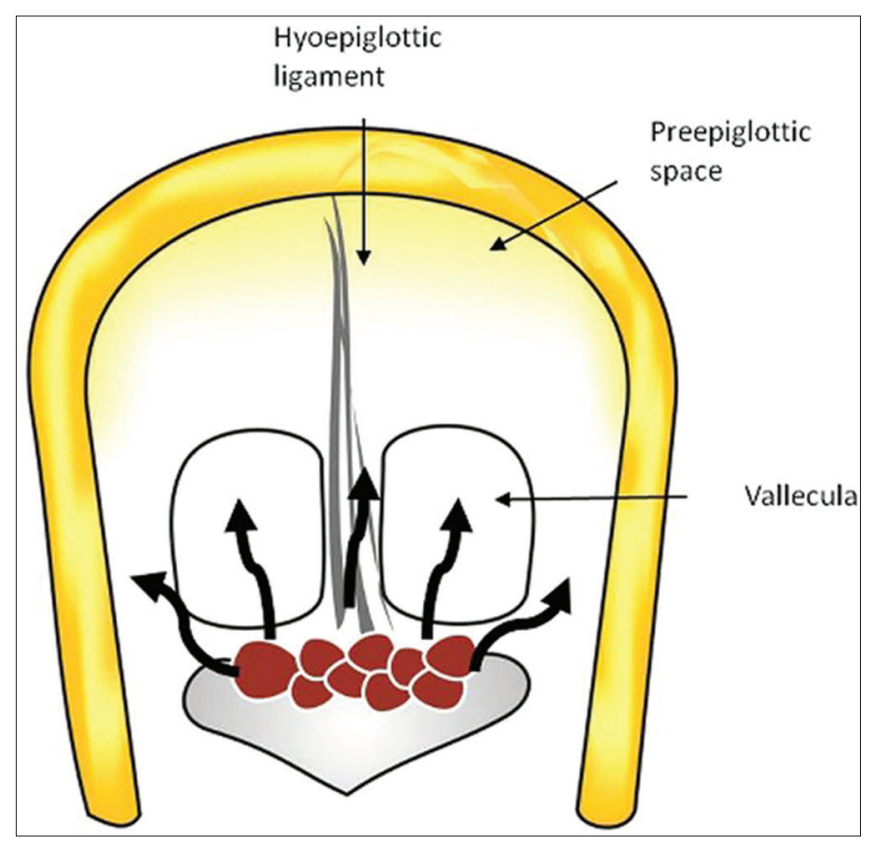

Figure 5B: Supraglottic mass arising from the epiglottis. Axial line diagram shows the pathways of spread (thick black arrows) of epiglottic mass (red colour)

\section{c. False cord SCC}

These are lateral masses with a strong predilection for submucosal spread into the PGS [Figures 10 and 11] More extensive tumor may destroy the thyroid cartilage and spread transglottically into the glottis and subglottis. Tumor spread to the PGS on CT or MRI is seen as replacement of the normal paraglottic fat by the enhancing tumor tissue [Figures 11 and 18a]. Both CT and MRI have a high sensitivity of about $95 \%$ to detect paraglottic tumor spread, the specificity, however, ranges between 50 and $75 \%$ as peritumoral inflammation may mimic tumor resulting in false positive assessments. ${ }^{[7,9]}$

\section{Glottic SCC}

Glottic SCCs represent about $65 \%$ of all laryngeal cancers. Hoarseness of voice due to vocal cord involvement is the primary presenting symptom in these patients. Metastatic nodal disease is rare in glottic carcinomas due to the sparse lymphatic drainage of the glottis. The 5-year survival rate for T1 glottic cancers is $90 \%$ and falls to $25 \%$ for T4 cancers. ${ }^{[12]}$

Glottic SCCs commonly arise from the anterior half of the vocal cord and spread into the anterior commissure. Anterior commissural disease is seen on CT or MRI as soft tissue thickening of more than 1-2 $\mathrm{mm}$. The accuracy of $\mathrm{CT}$ in predicting anterior commissure involvement is about $75 \%{ }^{[14]}$

From the anterior commissure, the tumor may spread further anteriorly into the contralateral cord and the thyroid cartilage or posteriorly into the posterior commissure, 


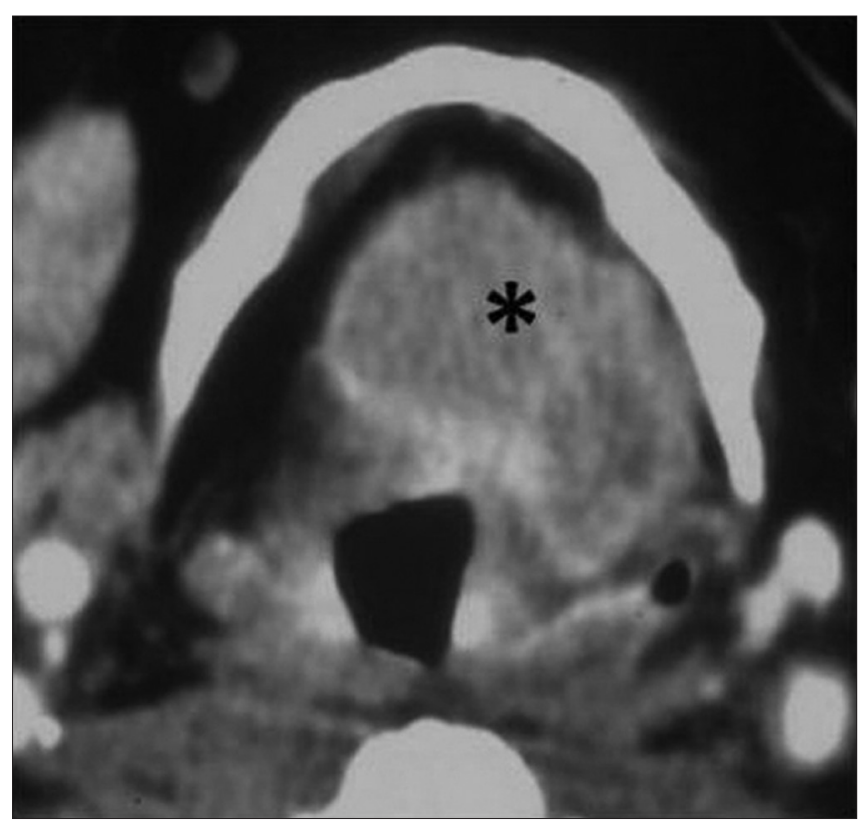

Figure 6: Supraglottic SCC - epiglottis. Axial contrast CT image shows a lobulated enhancing epiglottic mass filling the preepiglottic space (black asterisk)

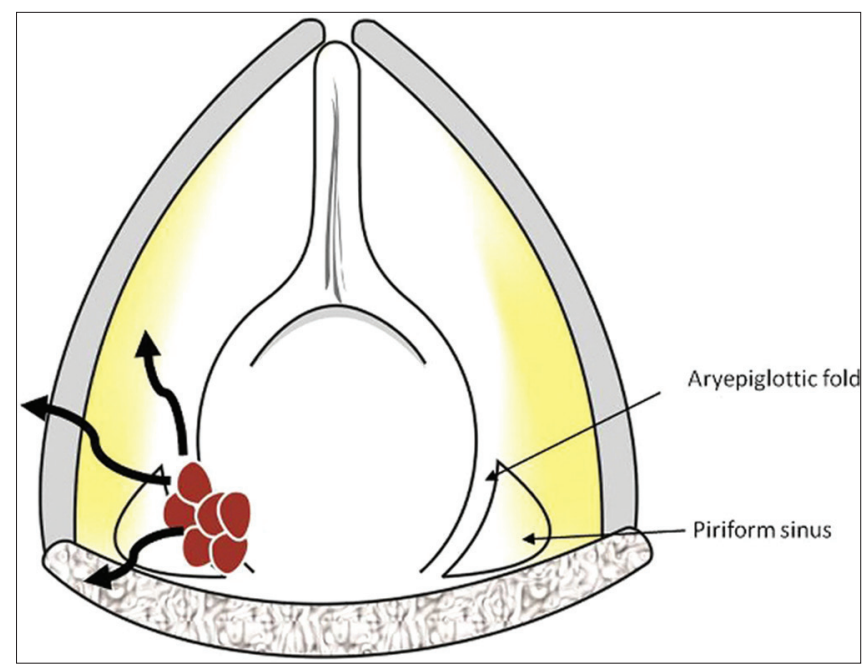

Figure 8: Supraglottic mass arising from the aryepiglottic fold. Line diagram shows a section through the aryepiglottic fold in the axial plane. The mass in the false cord is seen in red with pathways of spread in black curved arrows

the arytenoids, cricoarytenoid joint and the cricoid cartilage [Figures 12 and 13]. While vocal cord mobility is best assessed at endoscopy, disease in the cricoarytenoid joint and interarytenoid region [Figures 14A, B] have been described as imaging correlates for vocal cord fixation. ${ }^{[15]}$ The tumor may spread superiorly to access the PES and the PGS, or inferiorly to reach the subglottis. Subglottic spread below the anterior commissure is seen as an irregular thickening of the cricothyroid membrane. Tumor may gain access into the extralaryngeal tissues through the cricothyroid membrane [Figure 14].

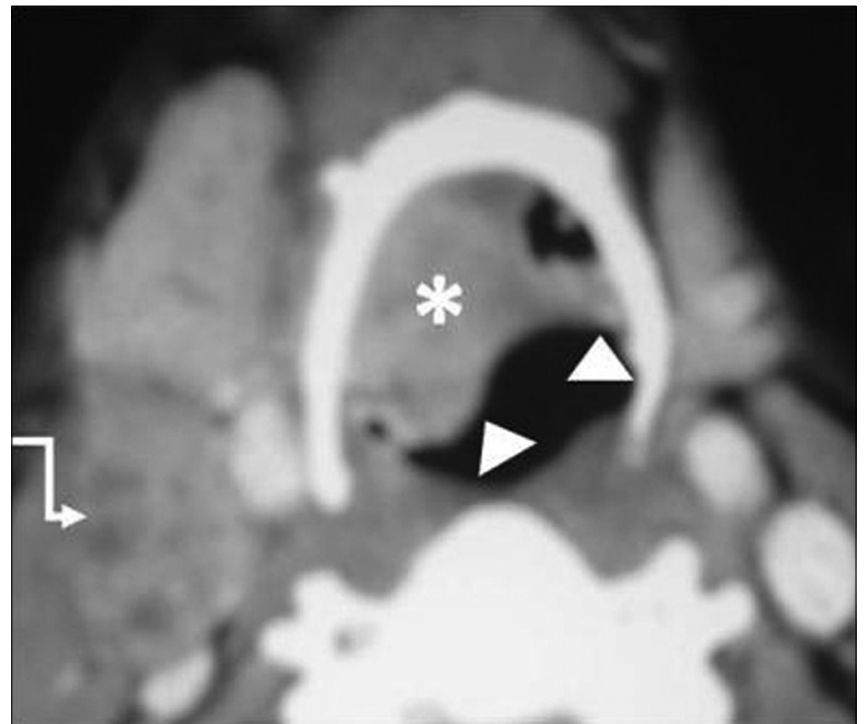

Figure 7: Supraglottic SCC - epiglottis. Axial contrast CT image in another patient shows the epiglottic mass (arrowheads) filling the right vallecula (white asterisk). Enlarged necrotic deep cervical node level II on the right side (elbow arrow)

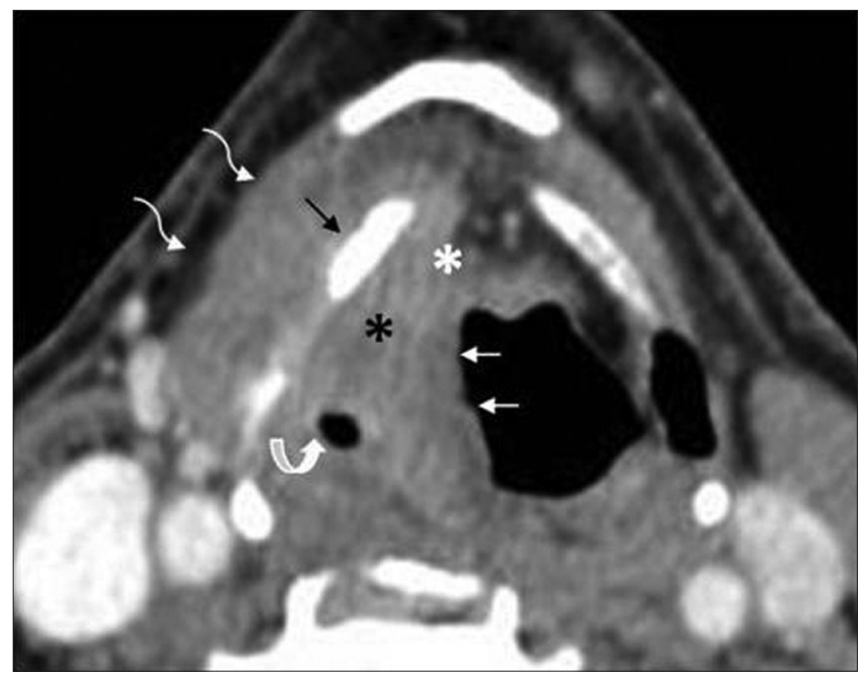

Figure 9: Supraglottic SCC - aryepiglottic fold. A right aryepiglottic fold mass (thin white arrows) is seen invading into preepiglottic (white asterisk) and right paraglottic space (black asterisk) and narrowing the right piriform sinus (curved white arrow). Note sclerosis of thyroid lamina (thin black arrow) with extralaryngeal tumor (white curved elbow arrows)

\section{Subglottic SCC}

These cancers are rare, accounting for only $5 \%$ of all laryngeal cancers, clinically silent and present late in the course of the disease and have a poor prognosis with a 5-year survival rate of $40 \%{ }^{\left[{ }^{[12]}\right.}$ Lymph node metastases are common and affect the pre and paratracheal nodes. Hence, the neck CT should be extended to include the superior mediastinum in patients with primary subglottic cancer.

Subglottic cancer is diagnosed if any tissue thickening is noted between the airway and the cricoids ring [Figure 15]. Due to their late presentation, invasion of the cricoids 


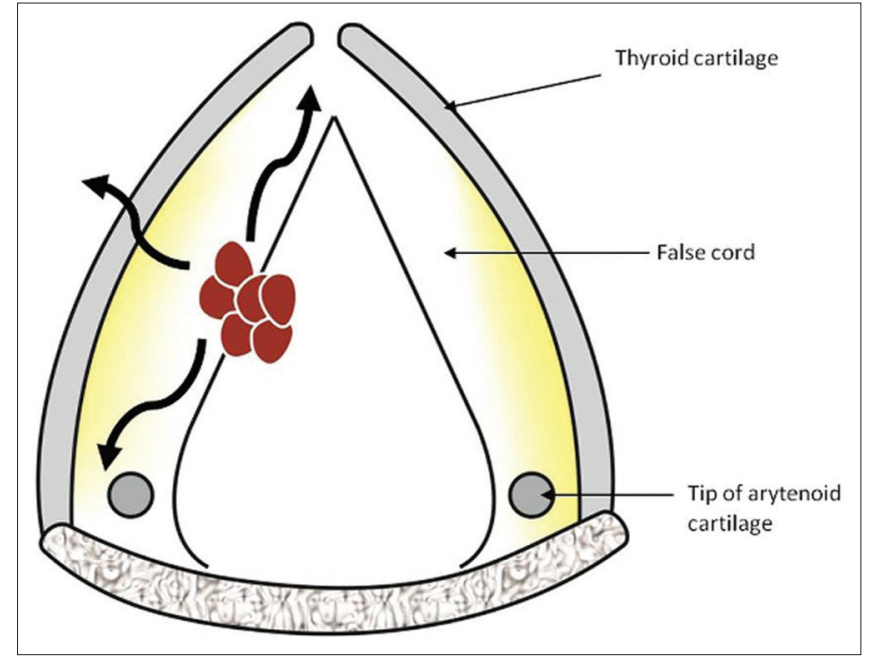

Figure 10: Supraglottic mass arising from the false cord. Line diagram shows a section through the false cord in the axial plane. The mass in the false cord is seen in red with pathways of spread in black curved arrows

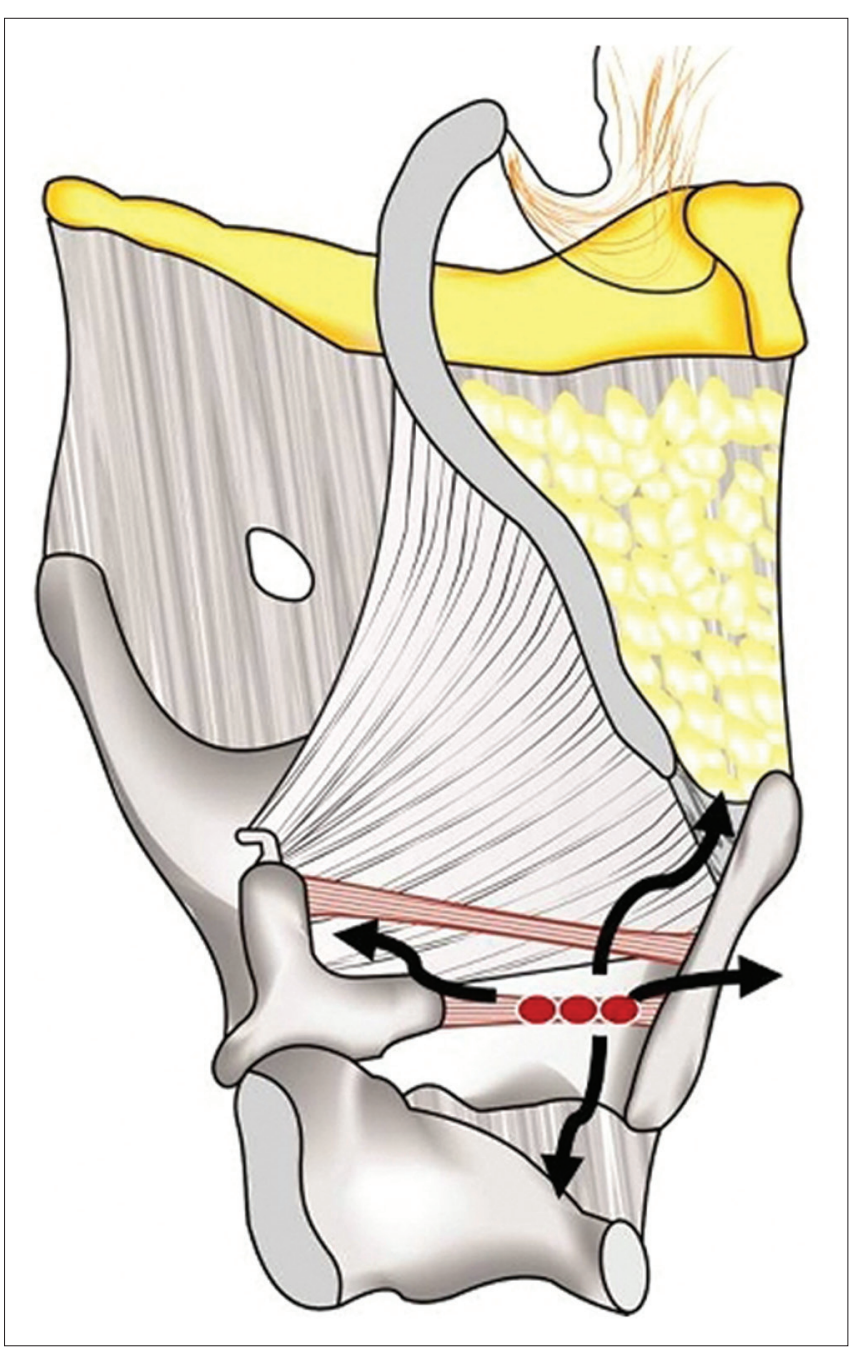

Figure 12A: Glottic mass. Sagittal line diagram shows the pathways of spread (thick black arrows) of glottic SCC (red mass lesion)

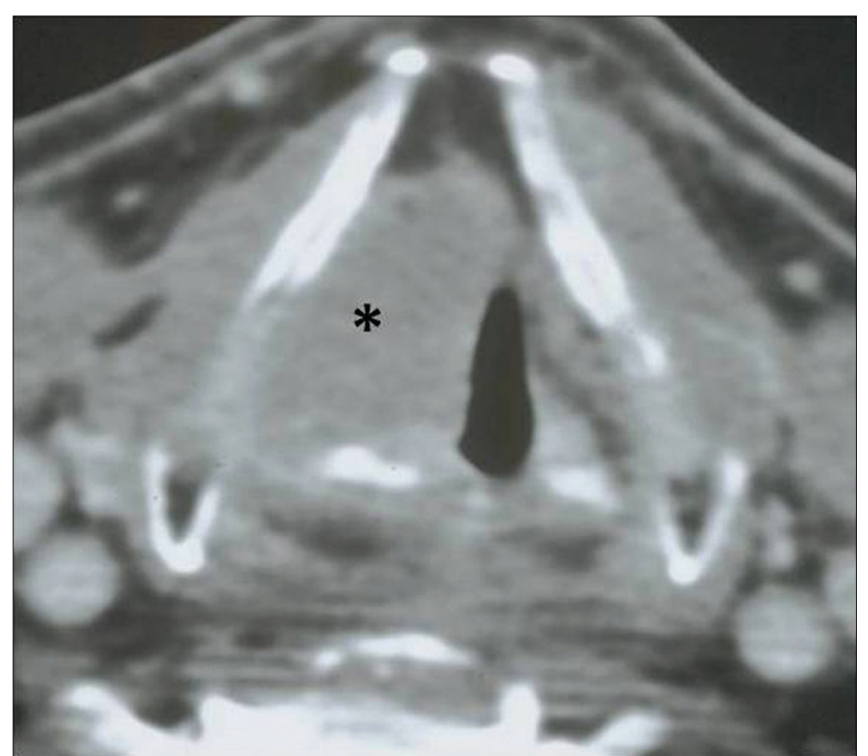

Figure 11: Supraglottic SCC - False cord. Axial contrast CT section through the false cords shows a mass within the right false cord and invading into the right PGS (black asterisk)

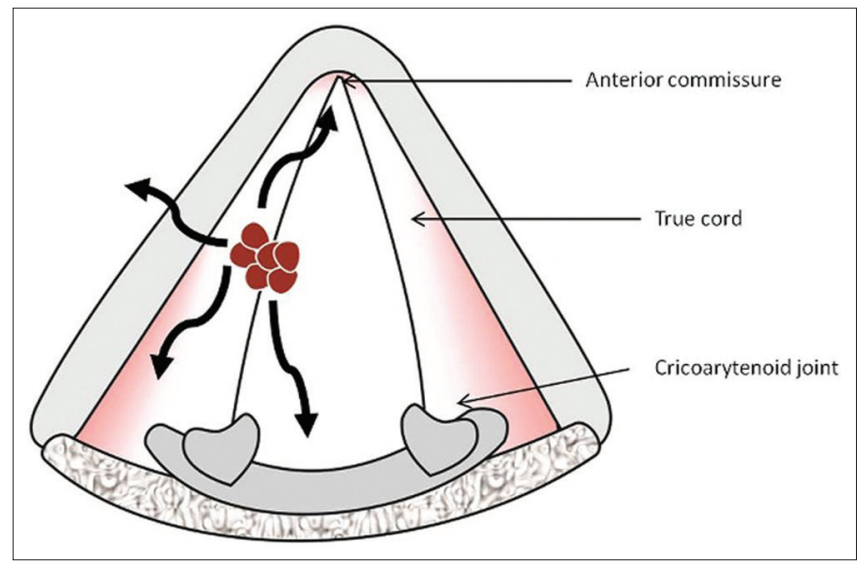

Figure 12B: Glottic mass. Axial line diagram shows the pathways of spread (thick black arrows) of glottic SCC (red mass lesion)

cartilage, trachea and the cervical esophagus with extralaryngeal spread are common findings in these patients at imaging [Figure 16].

\section{Transglottic SCC}

Laryngeal SCC encroaching on both, the glottis and supraglottis, with or without subglottic component and when the site of origin is unclear, is termed as transglottic tumor [Figure 17A, B] ${ }^{[6,7]}$ This tumor spread is frequently through the PGS and is readily identified on CT or MR imaging [Figure 18]. Transglottic carcinoma is frequently accompanied by metastatic lymphadenopathy. ${ }^{[7]}$ Coronal images are particularly helpful in assessing transglottic extension of tumor [Figure 18D].

\section{Influence of T-Staging on Treatment Options}

The treatment options for patients with laryngeal SCC 


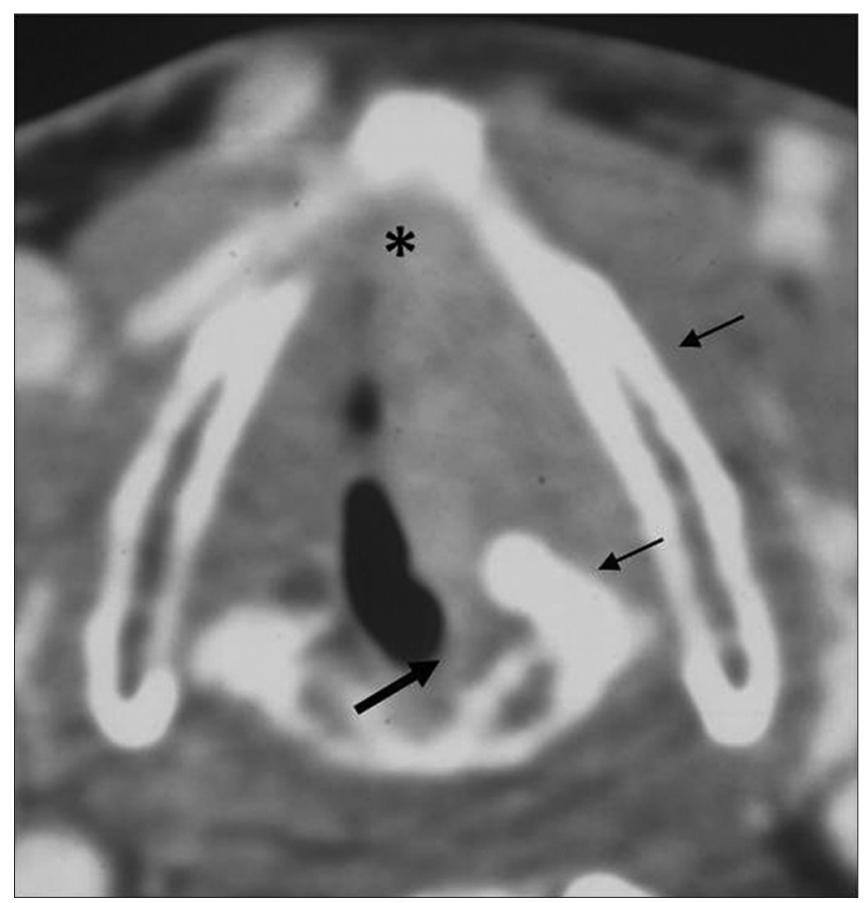

Figure 13: Glottic SCC. Axial contrast CT image shows a glottis mass in the left true cord reaching the anterior commissure (black asterisk). Mild thickening of posterior commissure is noted (thick black arrow) with sclerosis of left arytenoid and left lamina of thyroid cartilage

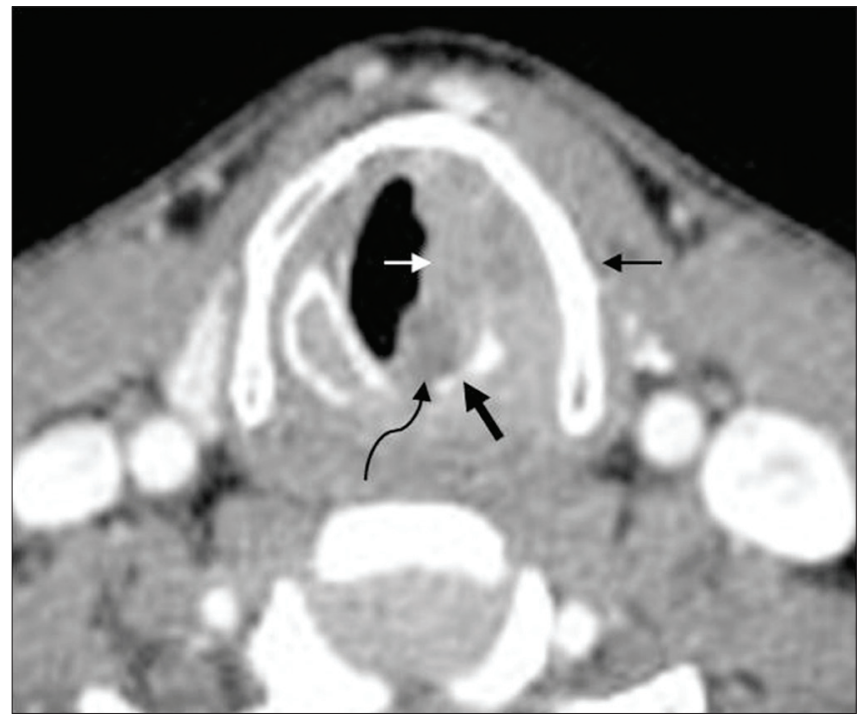

Figure 14B: Advanced glottic SCC. Axial contrast CT image at a caudal level shows the mass (thin white arrows) with disease in the posterior commissure (curved black arrow) and cricoid erosion (thick black arrow)

include primary radiation, surgery, chemotherapy and combinations of these. Surgical treatments include endoscopic laser resections, partial laryngectomies and total laryngectomy.

Important parameters for T-staging that influence the management in patients with laryngeal cancer include presence of tumor in the laryngeal submucosal spaces, spread across the commissures, cartilage invasion,

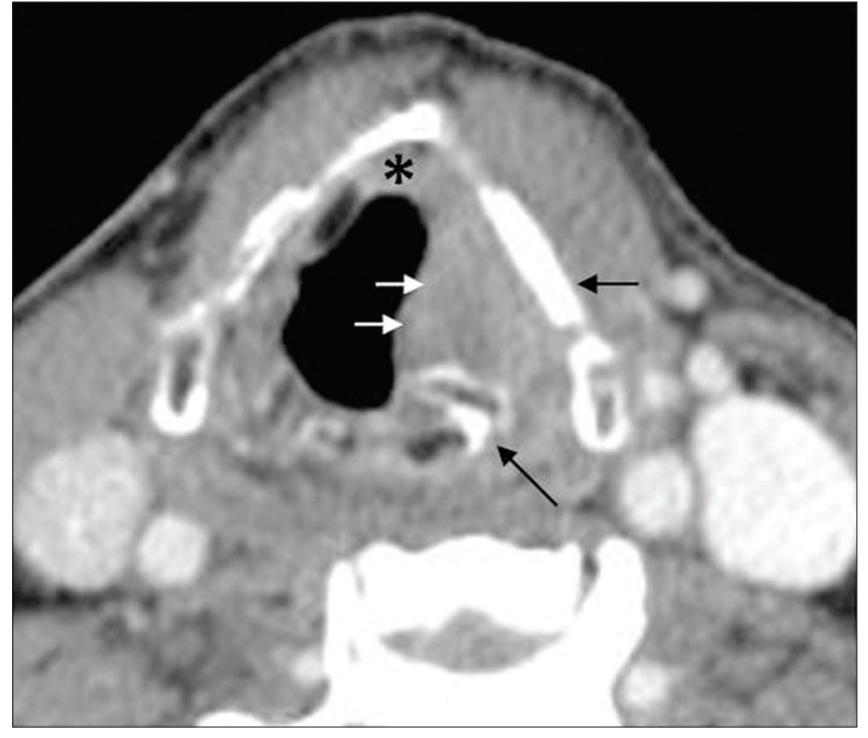

Figure 14A: Advanced glottic SCC. Axial contrast CT image shows aleft vocal cord mass (thin white arrows) reaching anterior commissure (asterisk). Note the sclerosis of left thyroid lamina and left cricoarytenoid joint (thin black arrows)

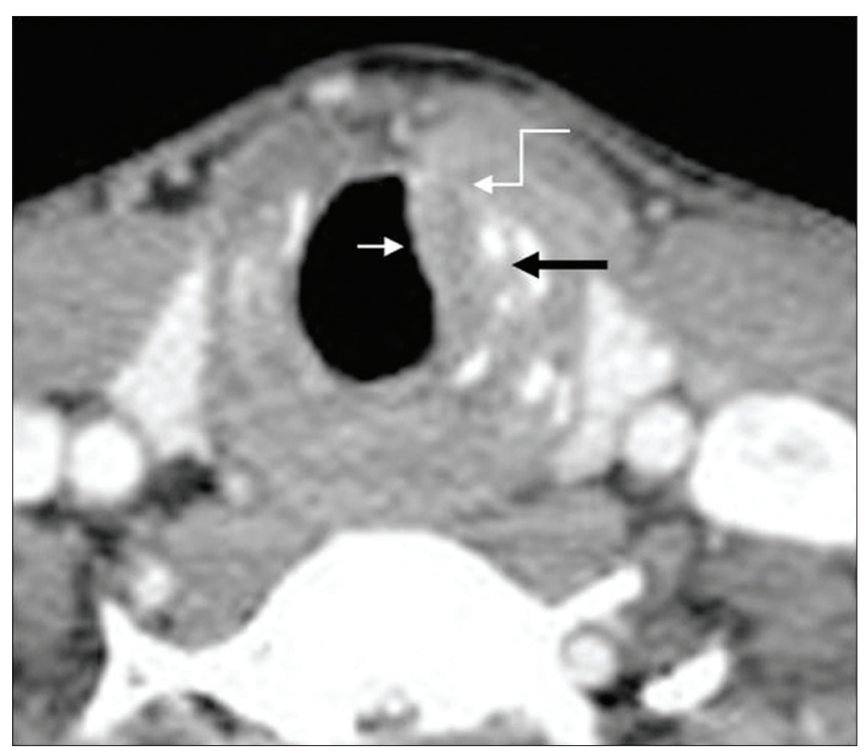

Figure 14C: Advanced glottic SCC. Axial contrast CT image through the subglottis shows the mass extending into the subglottis (thin white arrow) with irregularity of the cricothyroid membrane and extralaryngeal spread (white elbow arrow)

transglottic, deep subglottic and extralaryngeal extension. These are clinical blindspots and can be assessed on imaging studies alone.

Tumor in the laryngeal submucosal spaces is associated with an increased incidence of nodal metastases and a high risk of recurrence following radiation therapy. Bulky disease in the PES and PGS, transglottic and deep subglottic extension are negative indicators for primary radiotherapy and partial laryngectomy procedures. ${ }^{[4,7]}$ It is of prime importance to ascertain the inferior margin of a supraglottic mass. Only 


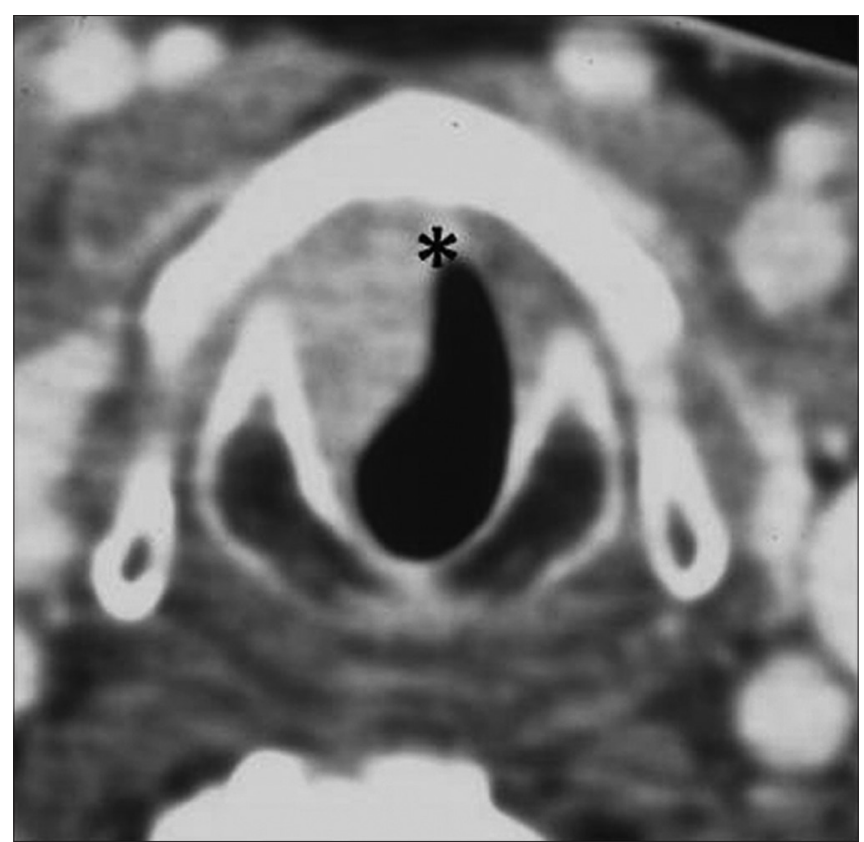

Figure 15: Subglottic SCC. Axial contrast CT image through the subglottis shows a smooth well-defined enhancing mass is seen on the right side (thin white arrows) reaching anteriorly just below the anterior commissure (black asterisk)

if the tumor is restricted to the supraglottis and does not involve the laryngeal ventricle or the arytenoids, is a partial laryngectomy indicated [Figures 19A, B]. Extension of glottis carcinoma across the anterior commissure to involve greater than $1 / 3$ of the contralateral vocal cord contraindicates a vertical hemilaryngectomy. Disease in the interarytenoid region and the posterior commissure precludes supracricoid laryngectomy. ${ }^{[, 7]}$ Presence of significant cartilage invasion on CT or MRI is also associated with a higher risk of tumor recurrence, increased risk of late complications and is predictive of poor response to radiation therapy. ${ }^{[7]}$ Isolated sclerosis of arytenoids cartilage at CT does not affect the radiation outcomes in patients with laryngeal carcinoma. ${ }^{[16]}$ Cricoid cartilage invasion always requires a total laryngectomy.

\section{T-staging Parameters Used for Prognosis of the Disease Process}

\section{Cartilage invasion}

MRI has a high sensitivity (89\%-95\%) but lower specificity $(74 \%-84 \%)$ as compared to $\mathrm{CT}$ for the detection of cartilage invasion. The negative predictive value of MRI to exclude cartilage invasion is also very high, at around $94 \%-96 \% .^{[17,18]}$

Presence of tumor invasion can be readily identified on the T1-weighted images if the cartilage is ossified [Figure 20A]. Tumor is seen as abnormal soft tissue intensity within the bright signal of the medullary fat of the cartilage. High intracartilaginous signal on fat-suppressed $\mathrm{T} 2$ weighted and cartilaginous enhancement on postcontrast fat-suppressed

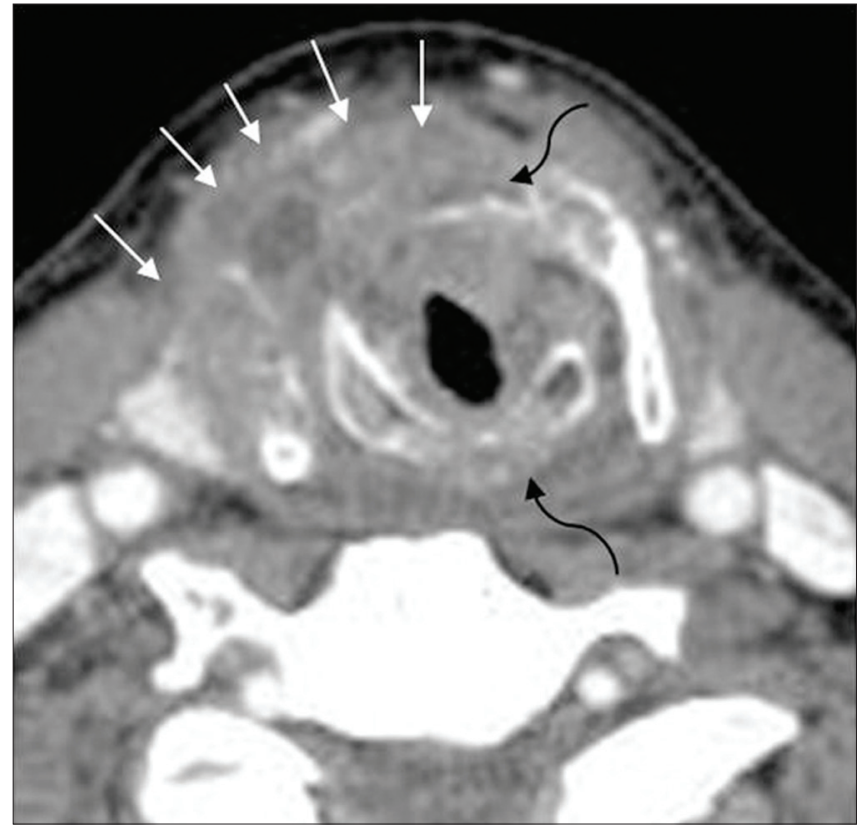

Figure 16: Advanced subglottic SCC. Axial CT image through the subglottis in another patient shows a circumferential subglottic mass with destruction of the cricoid and the thyroid cartilages (curved black elbow arrows) and extralaryngeal spread of tumor (thin white arrows)

T1-weighted sequences have been the accepted criteria for positive identification of neoplastic cartilaginous invasion [Figures 20B and 21]. However, peritumoral inflammation may mimic neoplastic invasion if these criteria are used, especially in the thyroid cartilage, thereby leading to false positive assessments. Recently, Becker, et al, ${ }^{[19]}$ reported that in assessing neoplastic infiltration of laryngeal cartilage at MR imaging, the T2-weighted and gadolinium-enhanced T1-weighted signal intensity should be compared with the signal intensity of the adjacent tumor on the corresponding sequences. If the cartilage displays higher signal intensity than tumor, a diagnosis of peritumoral inflammation within the cartilage is suggested; if, however, the cartilage displays a similar signal intensity to tumor, neoplastic cartilage invasion is suggested [Figures 20B and 21]. ${ }^{[18]}$

The CT criteria for reporting cartilage invasion include sclerosis, erosion, lysis and the presence of extralaryngeal tumor [Figures 22A, B].$^{[19,20]}$ While sclerosis has a high sensitivity $(83 \%)$ to detect intracartilaginous disease, it has a specificity that varies from one cartilage to another, being lowest in the thyroid cartilage $(40 \%)$ and higher in the cricoid and arytenoid cartilages $(76 \%$ and $79 \%$ respectively). ${ }^{[17-19]}$ Erosion or lysis and extralaryngeal tumor are highly specific criteria (86\%-95\%) for neoplastic cartilage disease; however, their sensitivity ranges between $64 \%$ and $72 \%$ and $44 \%$, respectively, as they occur very late in the course of the disease ${ }^{[18]}$ By applying a combination of all the above criteria, the overall sensitivity of $\mathrm{CT}$ is as high as $91 \%$ with a negative predictive value of $95 \%$. 


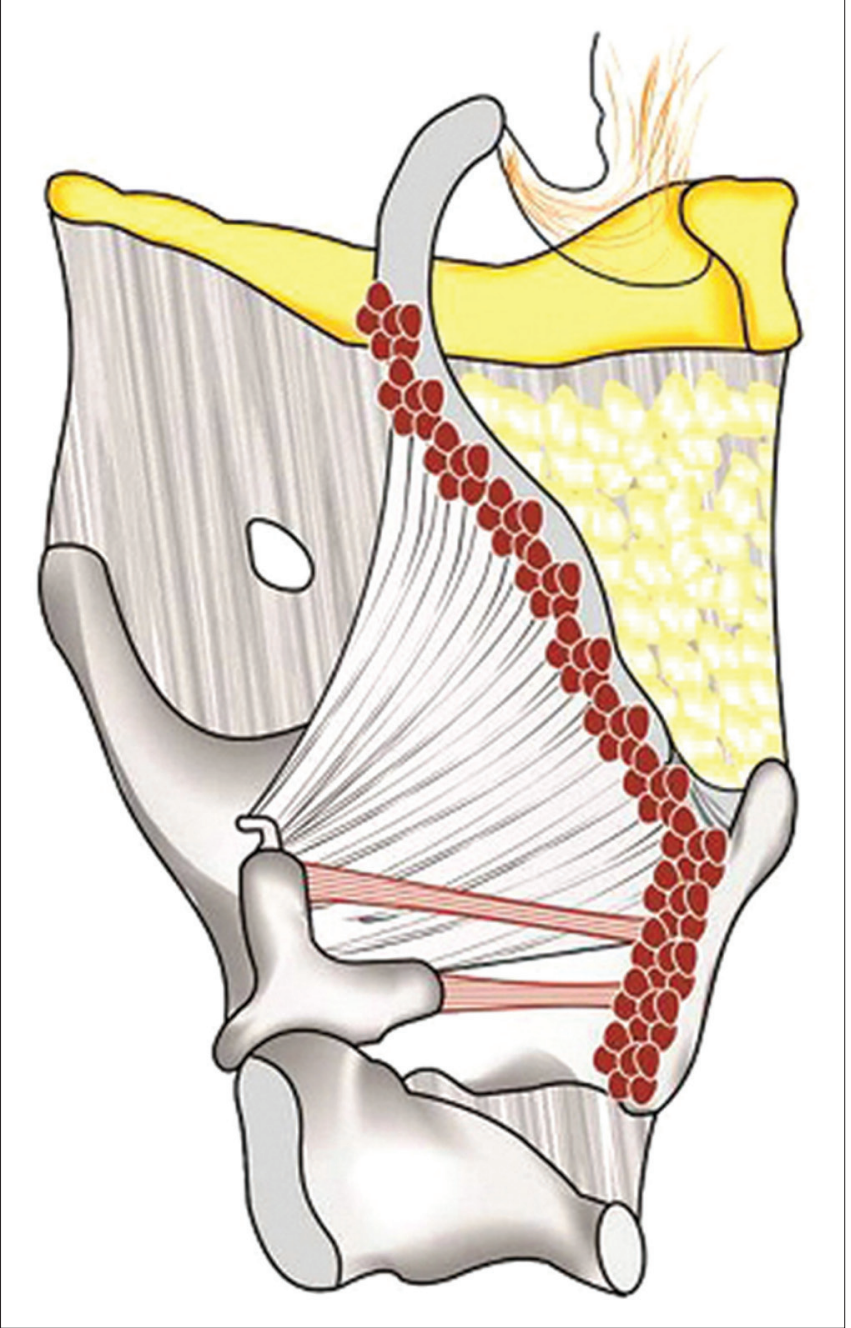

Figure 17A: Transglottic mass. Sagittal line diagram shows a large supraglottic mass (red colour) spanning the glottis and the subglottis

\section{Pretreatment tumor volume}

An increasing volume of the primary tumor correlates with an increasing rate of local failure. Supraglottic cancers with pretreatment CT volume of $\leq 6 \mathrm{ml}$ and glottic cancers with volumes $<3.5 \mathrm{ml}$ have shown better control rates ( $83 \%$ and $85 \%$, respectively), as compared to supraglottic cancers $>6 \mathrm{ml}$ and glottis cancers $>3.5 \mathrm{ml}(46 \%$ and $22 \%$, respectively). ${ }^{[21,22]}$ Abnormal signal intensity of laryngeal cartilages at MRI correlates with poor prognosis after radiation therapy if the tumor volume $>5 \mathrm{ml}$, but not if it is $<5 \mathrm{ml} .{ }^{[23]}$ Mukherji, et al. have found that the local surgical control rate for supraglottic tumors with volumes $<16 \mathrm{ml}$ was $98 \%$ compared with $40 \%$ for tumors with volumes $>16 \mathrm{ml} \cdot .^{[24,25]}$

\section{Nodal Disease (N Staging)}

A minimum axial diameter more than $10 \mathrm{~mm}$, round or spherical shape, a necrotic node of any size and a node

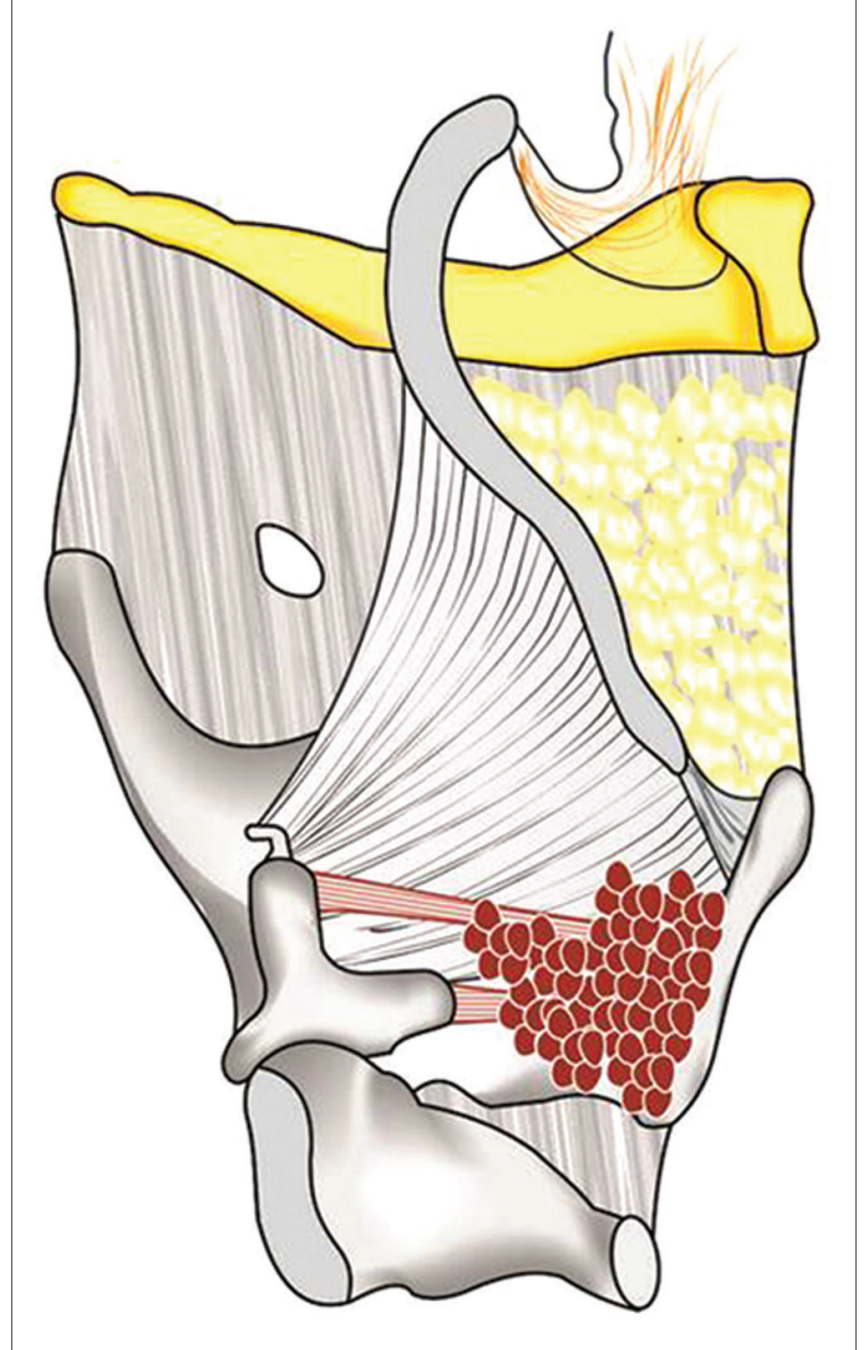

Figure 17B: Transglottic mass. Sagittal line diagram shows a transglottic mass (red colour) in the glottis extending into the supraglottis and subglottis

with indistinct spiculated margins (suggesting extranodal disease spread) are the generally accepted radiological criteria to diagnose malignant nodes at CT and MRI. ${ }^{[26,27]}$ The sensitivity and specificity of $\mathrm{CT}$ to detect nodal disease using these criteria are $90 \%$ and $75 \%$ respectively. The overall accuracy of PET-CT in identifying nodal disease is higher than that of CT alone, by almost $20 \%{ }^{[28]}$ However, PET-CT is not useful to exclude the presence of metastases in the clinically N0 neck. This is because $40 \%$ of metastatic lymph nodes are $<7 \mathrm{~mm}$ in diameter and PET-CT is not useful in evaluating small subcentimeter nodes. ${ }^{[29]}$ Standardized uptake values (SUV) of FDG uptake by the nodes has been used by some authors to identify nodal metastases;; ${ }^{[30]}$ however, to date, no universally accepted SUV threshold has been determined to differentiate benign from malignant nodal disease. Currently, ultrasonography (USG) with USG-guided fine needle aspiration cytology (FNAC) is the most accurate method for evaluating metastatic disease in subcentimeter size nodes ${ }^{[31]}$ Nodal staging is the most 


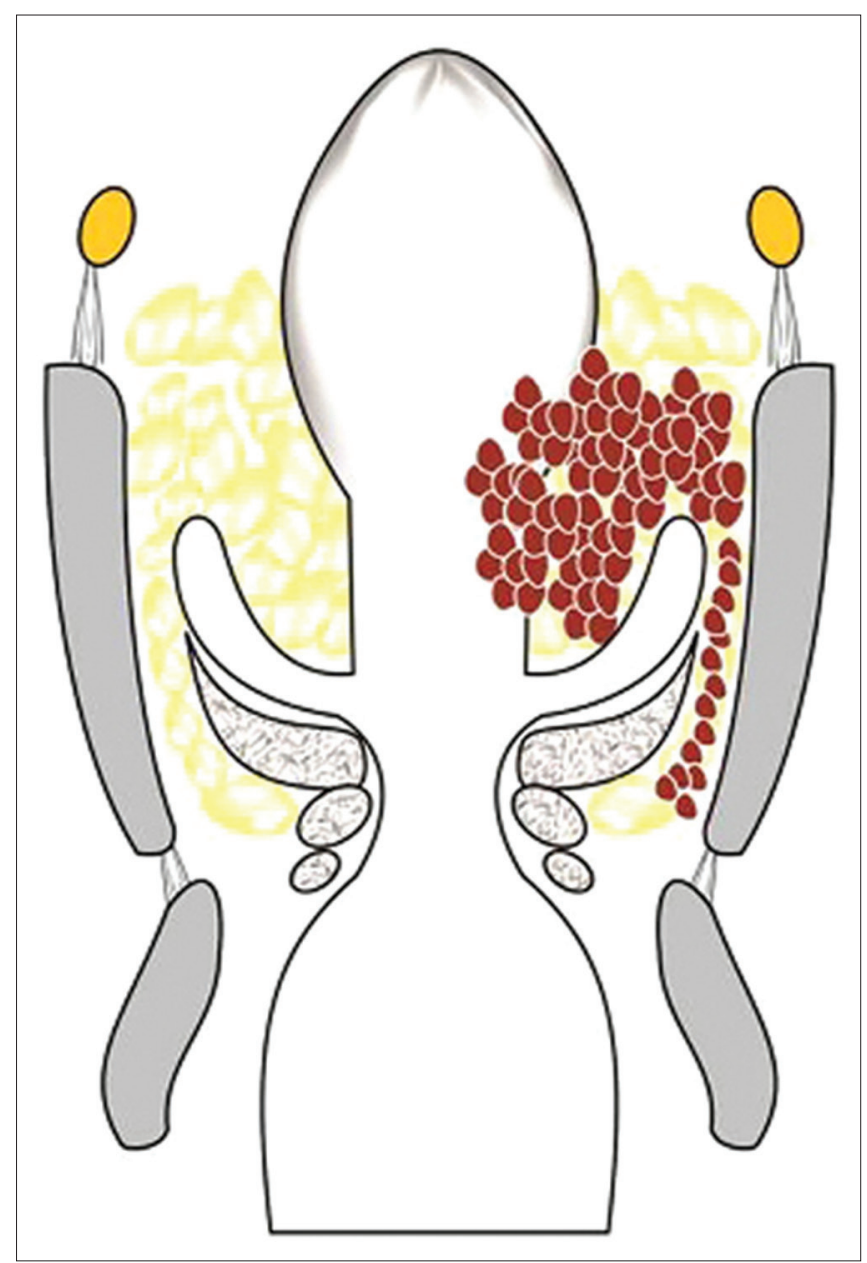

Figure 17C: Transglottic mass. Coronal line diagram shows the spread of transglottic cancer in the paraglottic space

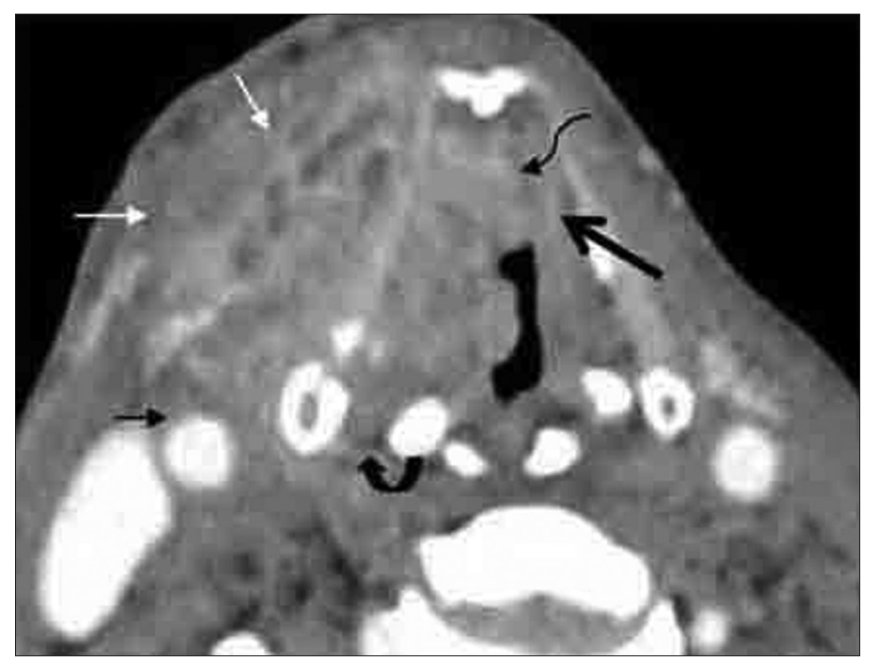

Figure 18B: Large transglottic SCC. Axial CT image shows tumor in right true cord, anterior commissure (curved black elbow arrow) and anterior left true cord (thick black arrow). Widened thyroarytenoid gap (curved black arrow), extralaryngeal tumor (thin white arrows) abutting right carotid artery (black arrow)

accurate prognostic factor for SCC. Unilateral nodal disease indicates a 50\% reduction in long term survival

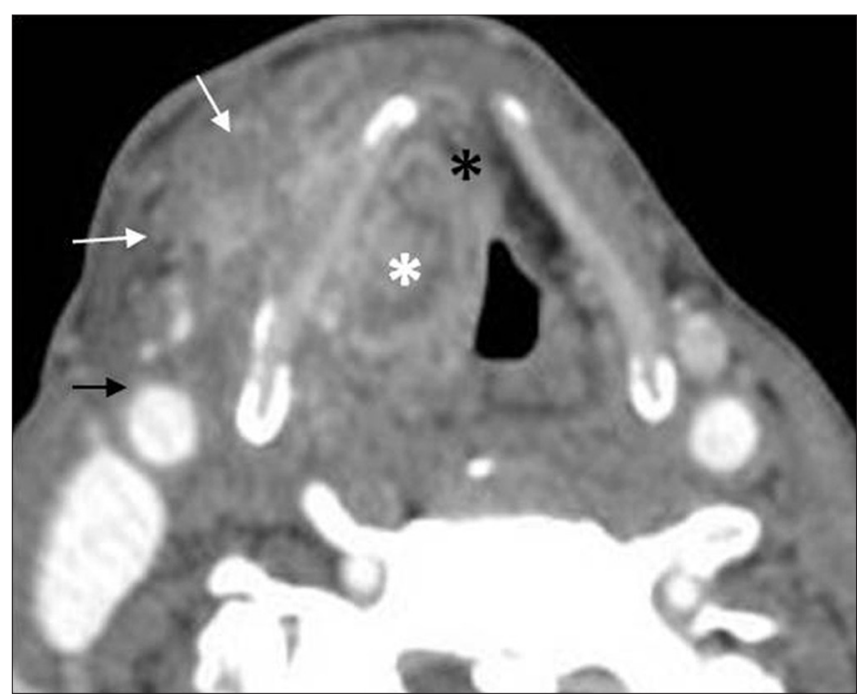

Figure 18A: Large transglottic SCC. Axial contrast CT image shows mass in the supraglottis invading the preepiglottic (black asterisk) and right paraglottic space (white asterisk). Extralaryngeal tumor (white arrows) is seen along outer aspect of right thyroid lamina abutting right carotid artery (black arrow)

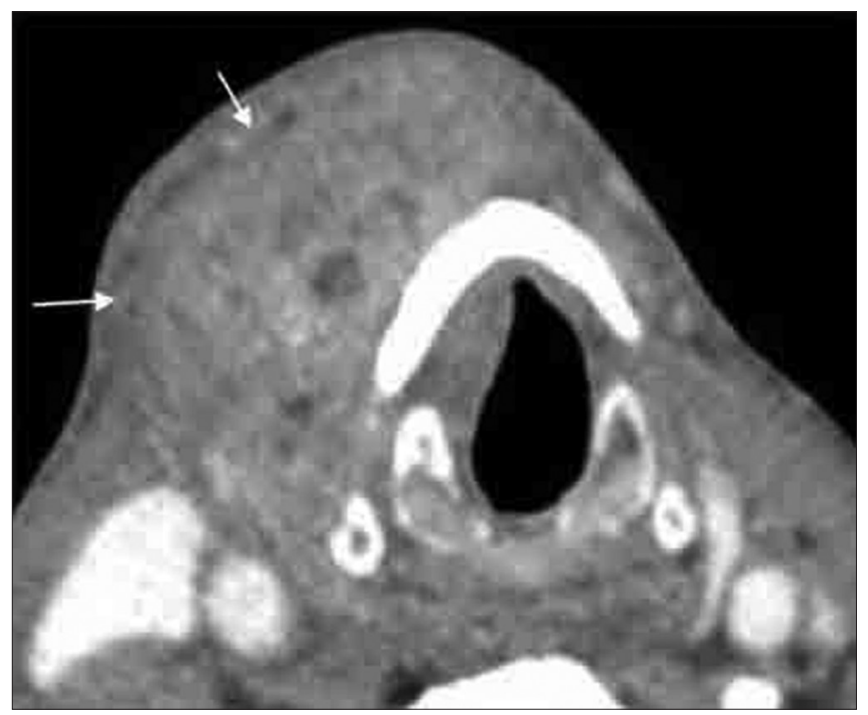

Figure 18C: Large transglottic SCC. Axial contrast CT image shows subglottic extension with extralaryngeal tumor (thin white arrows)

while a bilateral nodal involvement indicates a $75 \%$ reduction.$^{[8]}$ Additionally, extranodal spread increases the risk of treatment failure and increases the risk of recurrence by a factor of 10 and reduces the survival by $50 \% .^{[30,32]}$

\section{Systemic Metastases (M staging)}

The single most frequent site for distant metastases in laryngeal SCC is the lung, followed by bones and the abdomen. Systemic metastases are encountered in patients with advanced stage laryngeal SCC and upstage the disease from M0 to M1. While a chest radiograph may suffice in patients with early cancer, a contrast $\mathrm{CT}$ of the chest or whole body PET-CT is recommended in patients with advanced 


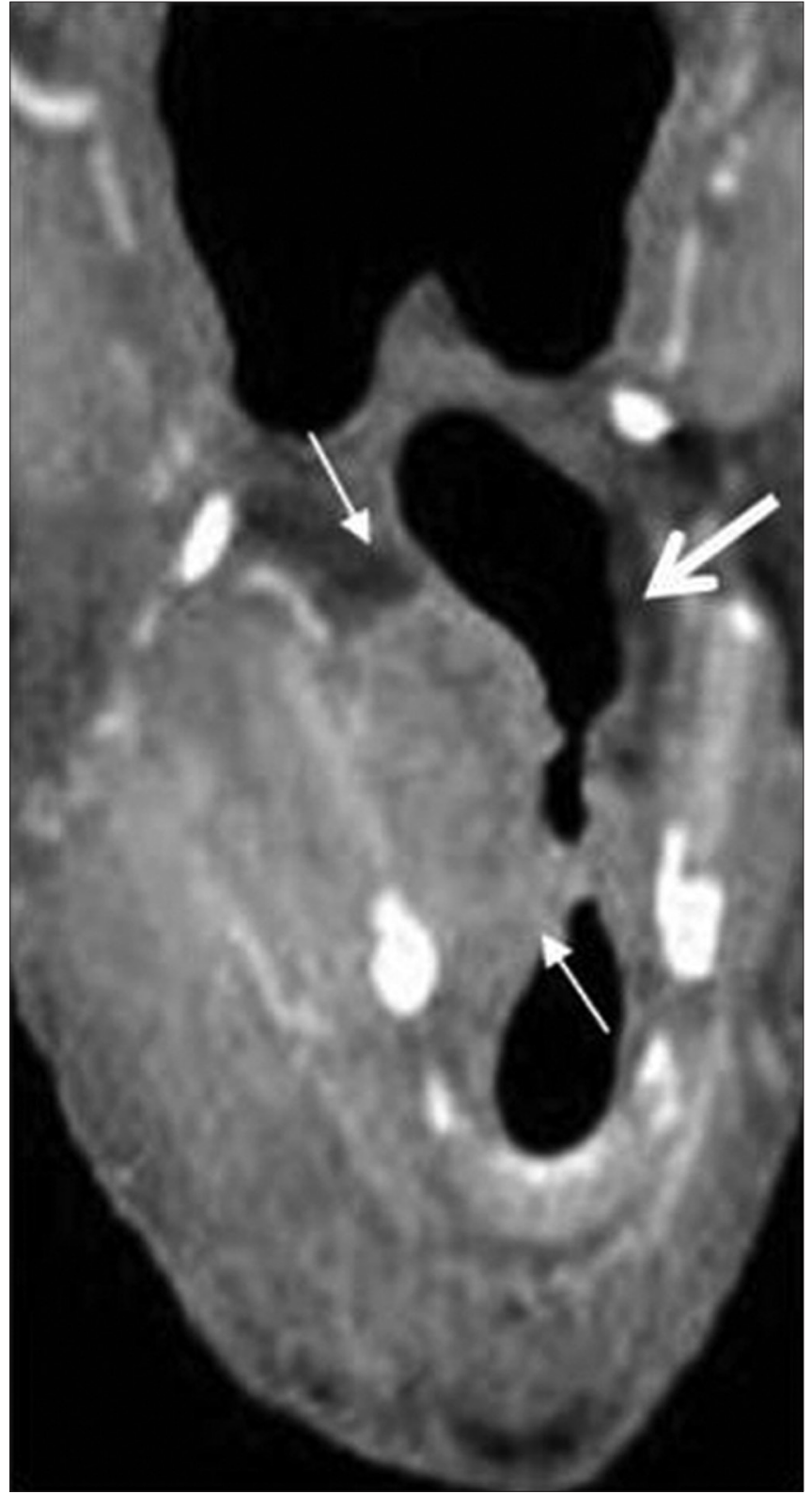

Figure 18D: Large transglottic SCC. Coronal CT shows the entire extent of transglottic mass spreading along the right paraglottic space (thin arrows). Note the normal left paraglottic fat space (thick arrow). Transglottic cancer precludes primary radiation or partial laryngectomy

laryngeal SCC. ${ }^{[30]}$ Presence of systemic metastases upstages the disease to M1 and precludes curative attempts by surgery.

\section{Post-Treatment Evaluation}

Post-treatment follow-up is critical in detecting the response to treatment and also for assessment of early recurrent disease. Surveillance is especially crucial in the first $2-3$ years because two-thirds of local recurrence and nodal metastases occur in this period.

Abaseline pretreatment FDG PET CT has been recommended

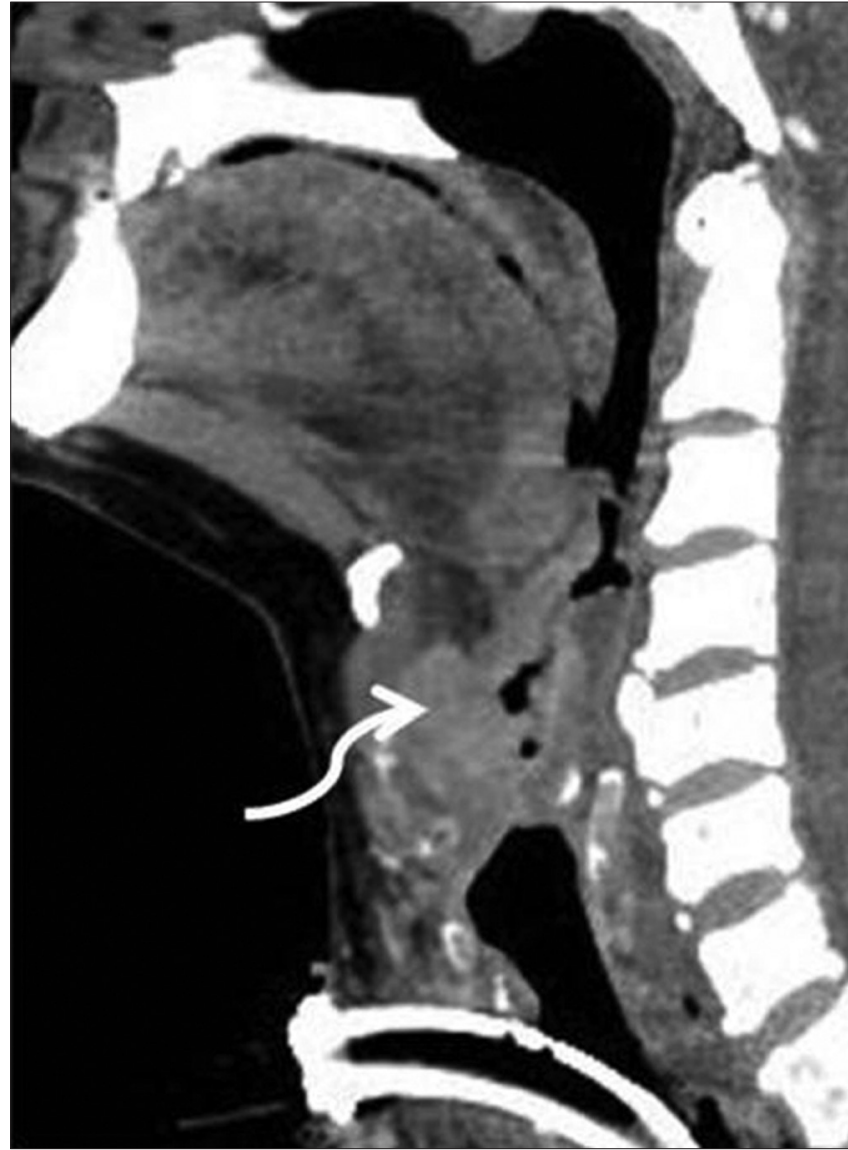

Figure 18E: Large transglottic SCC. Sagittal CT image very nicely shows tumor in the lower preepiglottic space (curved arrow)

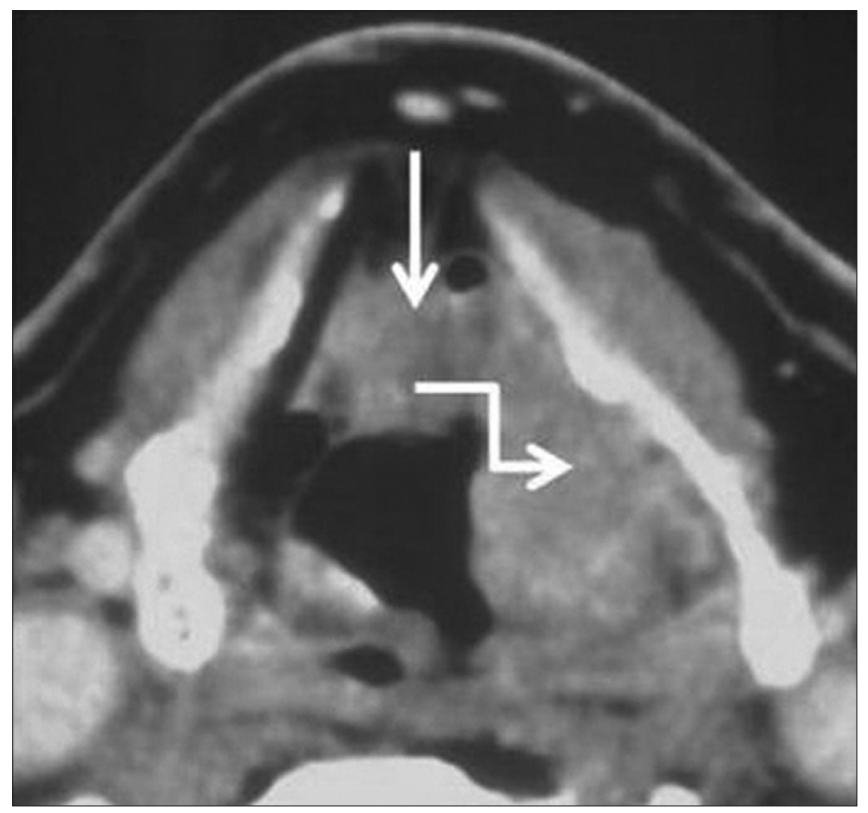

Figure 19A: Aryepiglottic fold SCC confined to supraglottis. Axial contrast CT image shows a left aryepiglottic fold mass infiltrating the preepiglottic (straight white arrow) and the left paraglottic space (white elbow arrow) 


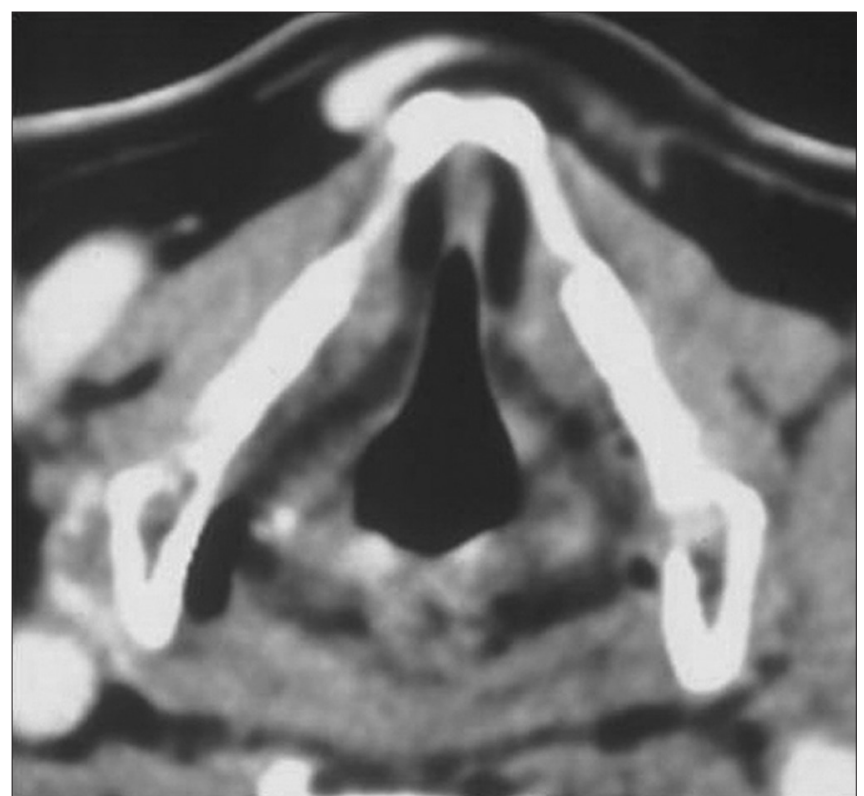

Figure 19B: Aryepiglottic fold SCC confined to supraglottis. Axial contrast CT image at the level of the false cord shows no tumor. Tumor confined to supraglottic larynx precluded the need for a total laryngectomy

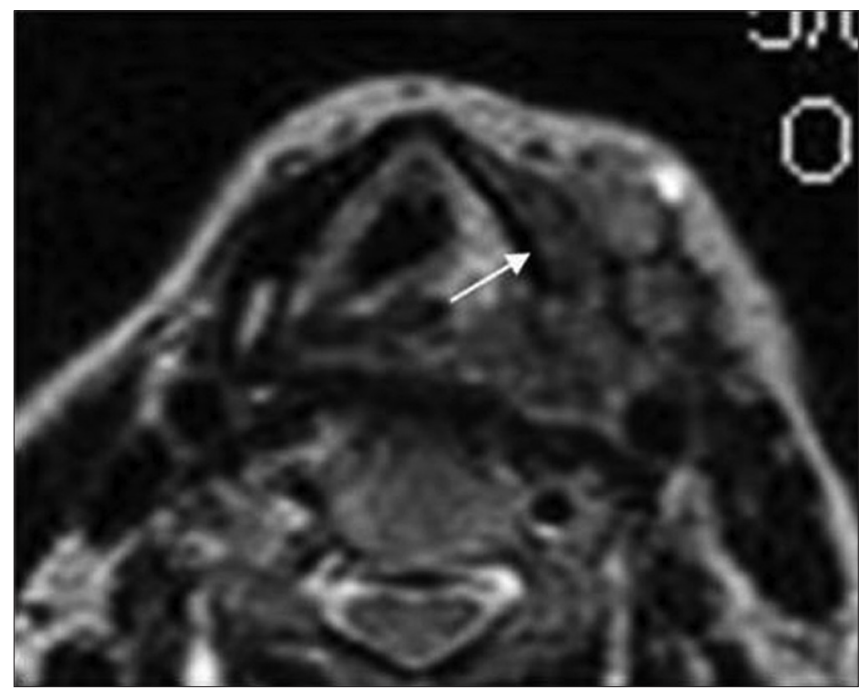

Figure 20B: Cartilage invasion on MRI. T2 axial image shows the large mass with cartilage destruction. The intracartilage signal is similar to the adjacent mass (thin arrow)

to use for comparison at subsequent post-treatment follow-up in patients with laryngeal SCC. A decreased FDG activity in the early phase of combined chemoradiation is associated with greater tumor response, survival and local control. ${ }^{[29]}$ A pretreatment SUV less than 9 in the primary tumor has been found to be predictive of a lower rate of local recurrence and improved disease free survival compared with a primary tumor SUV of 9 or more. ${ }^{[30]}$

While endoscopy remains the preferred method to diagnose mucosal recurrences, imaging contributes to the detection of deep recurrence. Surgery for laryngeal cancer

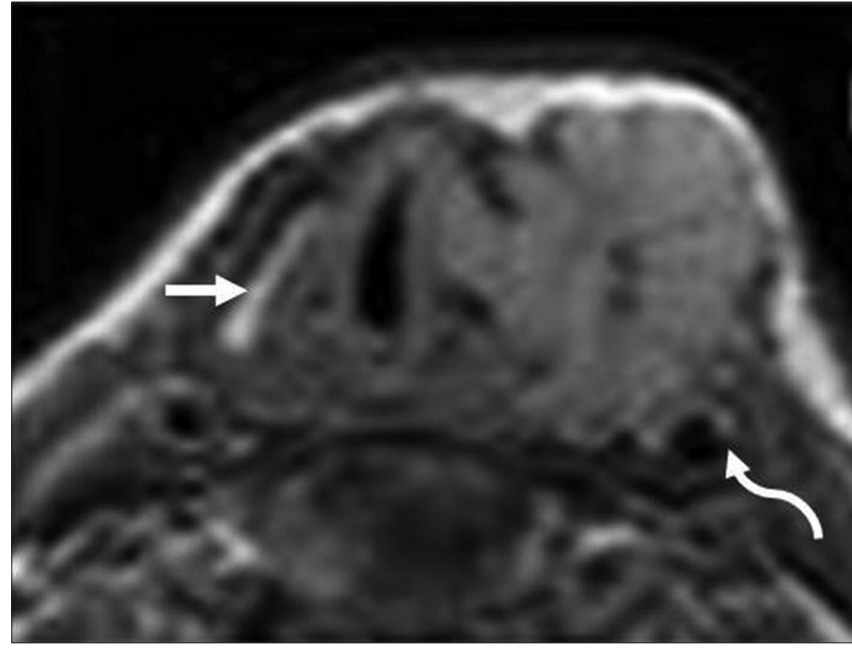

Figure 20A: Cartilage invasion on MRI. T1 axial image shows a large mass destroying left thyroid lamina with extralaryngeal spread encasing left carotid artery (curved arrow). See the normal ossified right thyroid lamina (thick arrow)

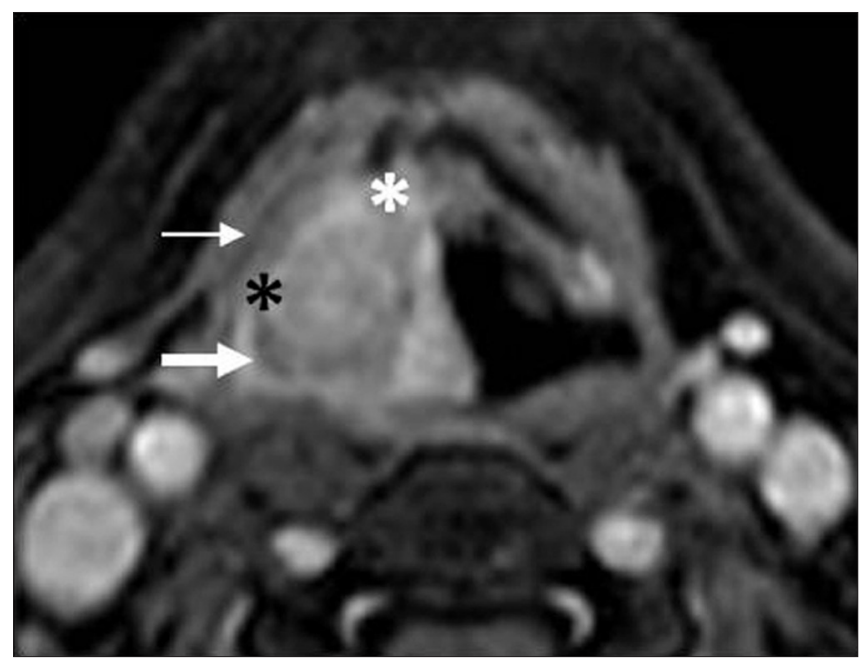

Figure 21: Cartilage invasion on MRI. Contrast fat-suppressed T1 image shows a large laryngohypopharyngeal mass invading preepiglottic (black asterisk), right paraglottic space (white asterisk) and right piriform sinus (thick arrow). Intracartilaginous enhancement is seen of similar intensity as tumor with thin rim of extralaryngeal tumor (thin arrow)

results in significant anatomic distortion making the diagnosis of recurrence extremely difficult. Recurrence following surgery is generally reported on CT, when focal areas of nodularity or soft tissue are noted in the surgical bed. These typically occur at the cut margins of the surgery where the tumor was previously located. Radiation for laryngeal SCC is followed by significant edema, thickening and abnormal enhancement in the laryngeal tissues. The normal fat in the PES and the PGS has a stippled appearance. The epiglottis, aryepiglottic folds and the arytenoids are swollen. Fragmentation, sclerosis and lysis of the cartilages may 


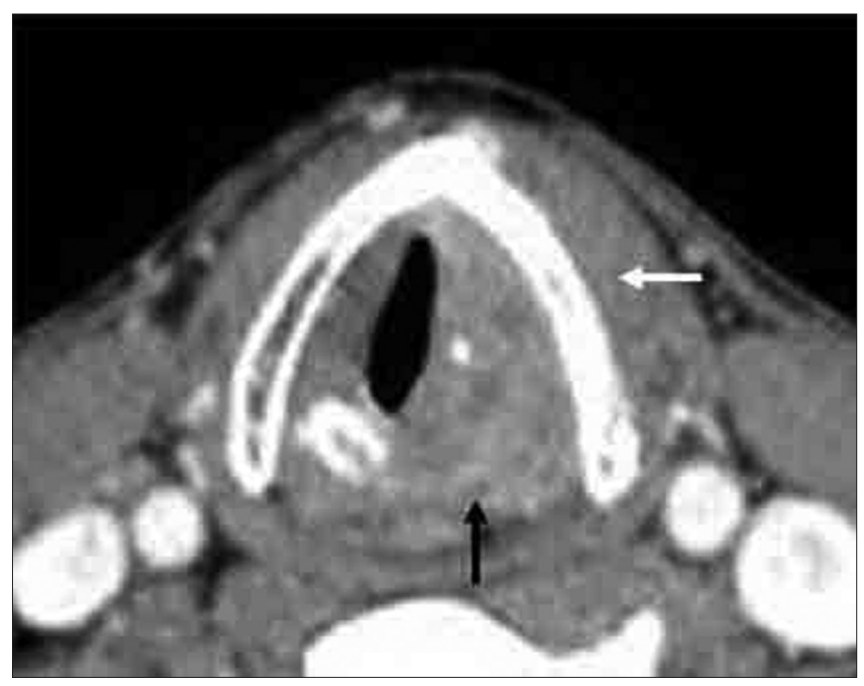

Figure 22A: Cartilage invasion on CT. Axial contrast CT image shows left true cord mass with left thyroid lamina sclerosis (white arrow) and cricoid destruction (black arrow)

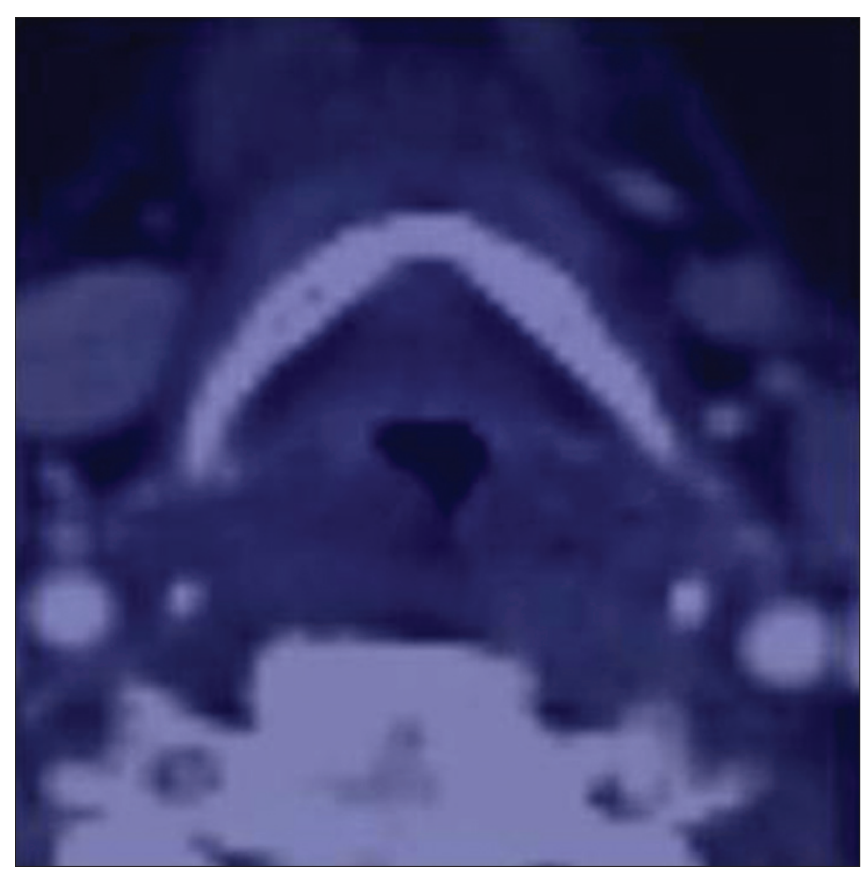

Figure 23A: Post-treatment CT following chemoradiation. Axial contrast CT image in a patient with epiglottic cancer, post chemoradiation therapy shows no obvious mass. The biopsy was negative

be seen suggesting the onset of chondronecrosis. While the knowledge of post-treatment larynx on CT and MRI scans helps in the interpretation of CT and MRI scans in these patients, PET-CT has superior diagnostic accuracy in detecting tumor recurrence [Figures 23A, B] ${ }^{[33-36]}$ The best results are obtained when PET-CT is performed 2-3 months after the completion of treatment. ${ }^{[35]}$ Successful radiation therapy leads to a substantial reduction of tumor volume within 4 months and treatment failure must be suspected if $50 \%$ or more of the tumor mass is still visible after this period. ${ }^{[37,38]}$

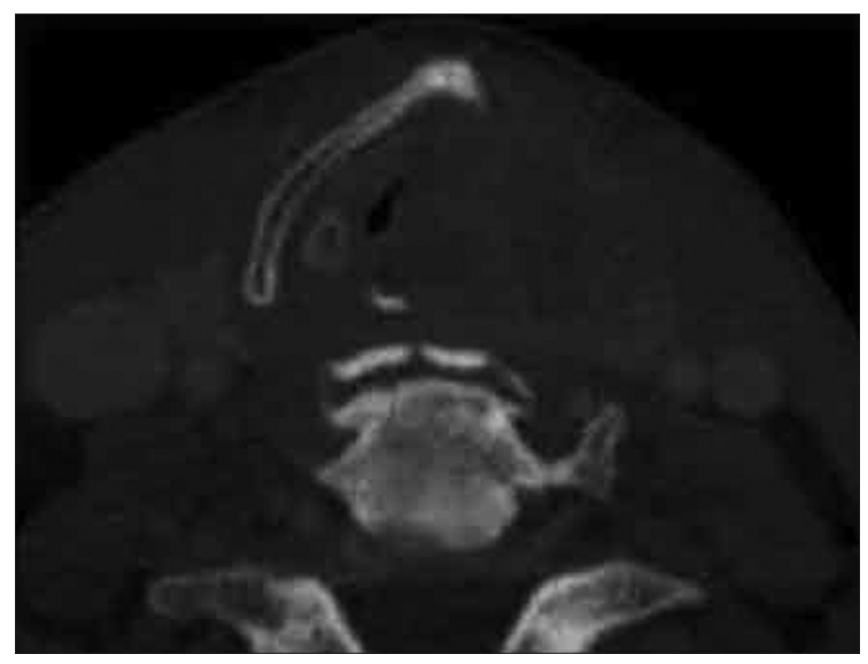

Figure 22B: Cartilage invasion on CT. Axial CT bone window image in another patient shows destroyed left thyroid lamina and left cricoarytenoid joint

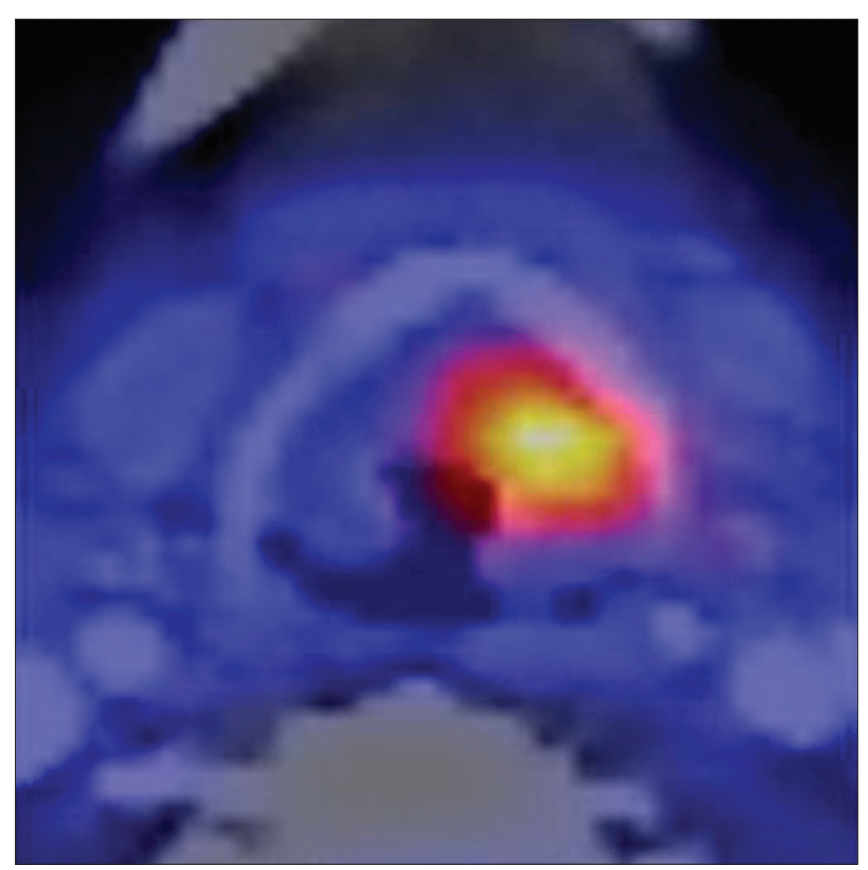

Figure 23B: Post-treatment PET-CT following chemoradiation. Axial PET-CT image obtained eight months later shows an area of uptake on the left side suggesting tumor recurrence. Biopsies were consistently negative

\section{Other Laryngeal Cancers}

While SCC is the commonest laryngeal cancer, the less common laryngeal cancers include the chondrosarcoma, lymphoma and the paraganglioma. These are usually submucosal and may not be seen at endoscopy except for a subtle bulge or asymmetry. The role of imaging in such tumors is to detect or confirm the presence of a submucosal mass, to define the deeper margins and to guide the endoscopist to the most appropriate site for biopsy. 


\section{Conclusions}

CT and MRI play a significant complementary role to clinical endoscopy in pretherapeutic staging of laryngeal SCC. Determination of the precise extent of cancer spread within the larynx (T staging) is the singlemost critical factor guiding treatment decisions in patients with localised laryngeal cancer. Additionally, imaging studies are routinely used to assess associated nodal disease ( $\mathrm{N}$ staging) and systemic metastases (M staging), presence of synchronous cancers and also post-therapeutic tumor recurrence in these patients. A clear understanding of the standard imaging techniques and protocols for imaging the larynx, and familiarity with the key anatomical features and characteristic patterns of tumor spread within the different regions of the larynx, are fundamental to the interpretation of CT and MRI scans of these patients.

\section{Acknowledgments}

The authors would like to thank Tutu Behera and Rachnoutsav Events for their help in making the schematic illustrations.

\section{References}

1. Parkin DM, Bray F, Ferlay J, Pisani P. Global cancer statistics 2002. CA Cancer J Clin 2005;55:74-108.

2. HashibeM, BoffettaP,ZaridzeD, ShanginaO,Szeszenia-DabrowskaN, Mates D, et al. Contribution of tobacco and alcohol to the high rates of squamous cell carcinoma of the supraglottis and glottis in central Europe. Am J Epidemiol 2007;165:814-20.

3. Nikolaou AC, Markou CD, Petridis DG, Daniilidis IC. Second primary neoplasms in patients with laryngeal carcinoma. Laryngoscope 2000;110:58-64.

4. Connor S. Laryngeal cancer: How does the radiologist help? Cancer Imaging 2007;7:93-103.

5. Hermans R. Staging of laryngeal and hypopharyngeal cancer. Eur Radiol 2006;16:2386-400.

6. Yousem DM, Tufano RP. Laryngeal Imaging. Magn Reson Clin N Am 2002;10:451-65.

7. Becker M, Burkhardt K, Dulguerov P, Allal A. Imaging of the larynx and the hypopharynx. Eur J Radiol 2008;66:460-79.

8. Curtin HD. The larynx. In: Som PM, Curtin HD, editors. Head and Neck Imaging. $4^{\text {th }}$ ed. St Louis, Mo: Mosby; 2003. p. 1595-699.

9. Zbaren P, Becker M, Läng H. Pretherapeutic staging of laryngeal carcinoma: Clinical findings, CT and MRI with histopathology. Cancer 1996;77:1263-73.

10. American Joint Committee on cancer: Larynx. In: Greene FL, editor. American Joint committee on cancer: AJCC staging manual. $7^{\text {th }}$ ed. NY: Springer; 2010. p. 57-68.

11. Castelijns JA, Hermans R, van den Brekel MW, Mukherji SK. Imaging of laryngeal Cancer. Semin Ultrasound CT MR 1998;19:492-503.

12. Chu MM, Kositwattanarerk A, Lee DJ, Makkar JS, Genden EM, Kao J, et al. FDG PET with contrast enhanced CT: A critical imaging tool for laryngeal carcinoma. Radiographics 2010;30:1353-72.

13. Loevner LA, Yousem DM, Montone KT, Weber R, Chalian AA, Weinstein GS. Can radiologists accurately predict PES invasion with MR imaging? AJR Am J Roentgenol 1997;169:1681-7.

14. Barbosa MM, Araújo VJ Jr, Boasquevisque E, Carvalho R,
Romano S, Lima RA, et al. Anterior vocal commissure invasion in laryngeal carcinoma diagnosis. Laryngoscope 2005;115:724-30.

15. Mancuso AA, Tamakawa Y, Hanafee WN. CT of the fixed vocal cord. AJR Am J Roentgenol 1980;135:7529-34.

16. Tart RP, Mukherji SK, Lee WR, Mancuso AA. Value of laryngeal cartilage sclerosis as a predictor of outcome in patients with stage T3 glottic cancer treated with radiation therapy. Radiology 1994;192:567-70.

17. Becker M, Zbären P, Casselman JW, Kohler R, Dulguerov P, Becker CD. Neoplastic invasion of laryngeal cartilage: Reassessment of criteria for diagnosis at MR imaging. Radiology 2008;249:551-9.

18. Zbaren P, Becker M, Lang H. Pretherapeutic staging of laryngeal carcinoma, Clinical findings, CT, and MRI compared with histopathology. Cancer 1996;77:1263-73.

19. Becker M, Zbären P, Delavelle J, Kurt AM, Egger C, Rüfenacht DA, et al. Neoplastic invasion of laryngeal cartilage: Reassessment of criteria for diagnosis at CT. Radiology 1997;203:521-32.

20. Beitler JJ, Muller S, Grist WJ, Corey A, Klein AM, Johns MM, et al. Prognostic accuracy of CT findings for patients with laryngeal cancer undergoing laryngectomy. J Clin Oncol 2010;28:2318-22.

21. Freeman DE, Mancuso AA, Parsons JT, Mendenhall WM, Million RR. Irradiation alone for supraglottic larynx carcinoma: Can CT findings predict treatment results? Int J Radiat Oncol Biol Phys 1990;19:485-90.

22. Lee WR, Mancuso AA, Saleh EM, Mendenhall WM, Parsons JT, Million RR. Can pre-treatment CT findings predict local control in T3 SCC of the glottis treated with radiotherapy alone? Int J Radiat Oncol Biol Phys 1993;25:683-7.

23. Castelijns JA, van den Brekel MW, Tobi H, Smit EM, Golding RP, van Schaik C, et al. Laryngeal carcinoma after radiation therapy: Correlation of abnormal MR imaging signal patterns in laryngeal cartilage with the risk of recurrence. Radiology 1996;198:151-5.

24. Mukherji SK, O'Brien SM, Gerstle RJ, Weissler M, Shockley W, Castillo M. Tumor volume: An independent predictor of outcome for laryngeal cancer. J Comput Assist Tomogr 1999;23:50-4.

25. Mukherji SK, O’Brien SM, Gerstle RJ, Weissler M, Shockley W, Stone JA, et al. The ability of tumor volume to predict local control in surgically treated SCC of the supraglottic larynx. Head Neck 2000;22:282-7.

26. Castelijns JA, van den Brekel MW. Imaging of lymphadenopathy in the neck. Eur Radiol 2002;12:727-38.

27. Harnsberger HR. SCC: Nodal staging. Handbook of Head and Neck Imaging. $2^{\text {nd }}$ ed. St Louis, Mo: Mosby; 1994. p. 283-98.

28. Jeong HS, Baek CH, Son YI, Ki Chung M, Kyung Lee D, Young Choi J, et al. Use of integrated FDG PET-CT to improve the accuracy of initial cervical nodal evaluation in patients with head and neck SCC. Head Neck 2007;29:203-10.

29. Mukherji SK, Bradford CR. Controversies: Is there a role for PET CT in the initial staging of head and neck SCC. AJNR Am J Neuroradiol 2006;27:243-5.

30. Hafidh MA, Lacy PD, Hughes JP, Duffy G, Timon CV. Evaluation of the impact of addition of PET to CT and MR scanning in the staging of patients with HNSCC. Eur Arch Otorhinolaryngol 2006;263:853-9.

31. Richards PS, Peacock TE. The role of ultrasound in the detection of cervical lymph node metastases in clinically N0 squamous cell carcinoma of the head and neck. Cancer Imaging 2007;7:167-78.

32. Puri SK, Fan CY, Hanna E. Significance of extracapsular lymph node metastases in patients with HNSCC. Curr Opin Otolaryngol Head Neck Surg 2003;11:119-23.

33. Schwartz DL, Rajendran J, Yueh B, Coltrera MD, Leblanc M, Eary J, et al. FDG-PET prediction of head and neck squamous cell cancer outcomes. Arch Otolaryngol Head Neck Surg 2004;130:1907-12. 
34. Gordin A, Daitzchman M, Doweck I, Yefremov N, Golz A, Keidar Z, et al. FDG-PET CT imaging in patients with carcinoma of the larynx: Diagnostic accuracy and impact on clinical management. Laryngoscope 2006;116:273-8.

35. Andrade RS, Heron DE, Degirmenci B, Filho PA, Branstetter BF, Seethala RR, et al. Post treatment assessment of response using PET-CT for patients treated with definitive radiation therapy for head and neck cancers. Int J Radiat Oncol Biol Phys 2006;65:1315-22.

36. Mukherji SK, Weadock WJ. Imaging of post-treatment larynx. Eur J Radiol 2002;44:108-19.
37. Mukherji SK, Mancuso AA, Kotzur IM, Mendenhall WM, Kubilis PS, Tart RP, et al. Radiologic appearance of the irradiated larynx. Part 1. Expected changes. Radiology 1994;193:141-8.

38. Mukherji SK, Mancuso AA, Kotzur IM, Mendenhall WM, Kubilis PS, Tart RP, et al. Radiologic appearance of the irradiated larynx. Part 2. Primary site response. Radiology 1994;193:149-54.

Cite this article as: Joshi VM, Wadhwa V, Mukherji SK. Imaging in laryngeal cancers. Indian J Radiol Imaging 2012;22:209-26.

Source of Support: Nil, Conflict of Interest: None declared.

\section{Staying in touch with the journal}

1) Table of Contents (TOC) email alert Receive an email alert containing the TOC when a new complete issue of the journal is made available online. To register for TOC alerts go to www.ijri.org/signup.asp.

\section{2) RSS feeds}

Really Simple Syndication (RSS) helps you to get alerts on new publication right on your desktop without going to the journal's website. You need a software (e.g. RSSReader, Feed Demon, FeedReader, My Yahoo!, NewsGator and NewzCrawler) to get advantage of this tool. RSS feeds can also be read through FireFox or Microsoft Outlook 2007. Once any of these small (and mostly free) software is installed, add www.ijri.org/rssfeed.asp as one of the feeds. 